\title{
Keck II Laser Guide Star AO System and Performance with the TOPTICA/MPBC Laser
}

\author{
Jason C.Y. Chin ${ }^{1, *}$, Peter Wizinowich ${ }^{1}$, Ed Wetherell ${ }^{1}$, Scott Lilley ${ }^{1}$, Sylvain Cetre ${ }^{1}$, Sam Ragland ${ }^{1}$, \\ Drew Medeiros ${ }^{1}$, Kevin Tsubota ${ }^{1}$, Greg Doppmann ${ }^{1}$, Angel Otarola ${ }^{2}$, Kai Wei ${ }^{2}$ \\ ${ }^{1}$ W. M. Keck Observatory, 65-1120 Mamalahoa Hwy., Kamuela, HI 96743, USA; \\ jchin@keck.hawaii.edu; phone 1808 881-3510; fax 1808 885-4464; www.keckobservatory.org \\ ${ }^{2}$ Thirty Meter Telescope. Pasadena, CA 91105; www.tmt.org
}

\begin{abstract}
The Keck II Laser Guide Star (LGS) Adaptive Optics (AO) System was upgraded from a dye laser to a TOPTICA/MPBC Raman-Fibre Amplification (RFA) laser in December 2015. The W. M. Keck Observatory (WMKO) has been operating its AO system with a LGS for science since 2004 using a first generation $15 \mathrm{~W}$ dye laser. Using the latest diode pump laser technology, Raman amplification, and a well-tuned second harmonic generator (SHG), this Next Generation Laser (NGL) is able to produce a highly stable $589 \mathrm{~nm}$ laser beam with the required power, wavelength and mode quality. The beam's linear polarization and continuous wave format along with optical back pumping are designed to improve the sodium atom coupling efficiency over previously operated sodium-wavelength lasers. The efficiency and operability of the new laser has also been improved by reducing its required input power and cooling, size, and the manpower to operate and maintain it.

The new laser has been implemented on the telescope's elevation ring with its electronics installed on a new Nasmyth sub-platform, with the capacity to support up to three laser systems for future upgrades. The laser is projected from behind the telescope's secondary mirror using the recently implemented center launch system (CLS) to reduce LGS spot size. We will present the new laser system and its performance with respect to power, stability, wavelength, spot size, optical repumping, polarization, efficiency, and its return with respect to pointing alignment to the magnetic field. Preliminary LGSAO performance is presented with the system returning to science operations. We will also provide an update on current and future upgrades at the WMKO.
\end{abstract}

Keywords: adaptive optics, laser guide star, W. M. Keck Observatory

\section{INTRODUCTION}

The Keck II LGS AO system (Wizinowich et al. [1]) has been in operation since 2004 using a dye laser developed by Lawrence Livermore National Laboratory (Friedman et al. [2]). This first generation dye laser (DL) and its side launch propagation system (SLS) have been responsible for an estimated 14,000 hours of science operations. Results using this laser and the LGS AO system supported 246 refereed science papers through 2015 (Wizinowich [3]). In 2010, a second generation solid state laser was developed by Lockheed Martin Coherent Technology (Lee et al. [4]) and installed on the Keck I telescope. This laser used the sum frequency generation technique of mixing two laser wavelengths, $1064 \mathrm{~nm}$ and $1319 \mathrm{~nm}$, to generate the $589 \mathrm{~nm}$ sodium wavelength. Both of these lasers tend to require significant manpower and resources to maintain and operate. In the fall of 2014, Keck received a TOPTICA Photonics AG and MPB Communications (MPBC) laser (Friendenauer et al. [5]) using RFA. This laser was developed by a guide star consortium comprised of the European Southern Observatory (ESO), WMKO and the Thirty Meter Telescope with TOPTICA/MPBC as the contractors. This third generation laser was tested and integrated on the Keck II telescope in 2015 with the CLS. Commissioning was conducted in the first quarter of 2016 with the LGS AO system going back into science mode in April of 2016.

This third generation laser produces significantly more return than the previous dye and solid state lasers. The laser's intrinsic performance at the Maunakea site, Hawaii is examined in detailed, along with its operations model and maintenance. Challenges with the laser and the CLS are discussed and their impact on operations. The laser's on-sky performance is presented as compared to the previous two generations of laser with a focus on the dye laser. Since the new laser system has only been in science operation as of April 2016 only preliminary science data is provided. A quick look is provided of other recent $\mathrm{AO}$ upgrades and how they fit into the planned $\mathrm{AO}$ upgrades at the observatory.

Adaptive Optics Systems V, edited by Enrico Marchetti, Laird M. Close, Jean-Pierre Véran, Proc. of SPIE Vol. 9909, 99090S (c) 2016 SPIE · CCC code: $0277-786 X / 16 / \$ 18 \cdot$ doi: $10.1117 / 12.2233138$ 


\section{NGL SYSTEM OVERVIEW}

The Keck II NGL system design review was held in April 2013. The TOPTICA/MPBC laser reviews were conducted separately with the LGS consortium. The preliminary design was held one year later followed by the detailed design in November 2014. The NGL system builds on the center launch system's commissioning in the spring of 2014 (Chin et al. [6]). The CLS beam transport system was integrated on the Keck II telescope using the dye laser initially and then subsequently with the NGL. The Keck I laser also has a similar CLS type beam transport, although more challenging due to the fixed gravity orientation of the laser mounted on the Nasmyth platform. The Keck I LGSAO system was completed in 2012 (Chin et al. [7]). The CLS reduces the spot elongation by projecting the laser beam from behind the telescope's secondary mirror. A negative impact of the CLS, as compared to the side launch system, is a throughput loss due to 19 additional optical elements in the Keck II beam transport system.

The NGL project's primary objective was to replace the DL with a TOPTICA/MPBC laser and integrate the necessary subsystems and infrastructure to support the new laser. The subsystems include a new platform on the telescope for the laser, infrastructure systems (power and cooling), an upgraded safety system, mechanical mounting and an optics bench to steer the laser into the beam train, motion control electronics, and software. The NGL system was integrated with the existing LGSAO system and instruments. The commissioning and prime science camera is the Near Infrared Camera II (NIRC2). For future considerations, the system was also designed to include all infrastructures necessary to support two additional TOPTICA/MPBC lasers as part of the Keck's proposed Next Generation Adaptive Optics System (NGAO; Wizinowich et al. [8]).
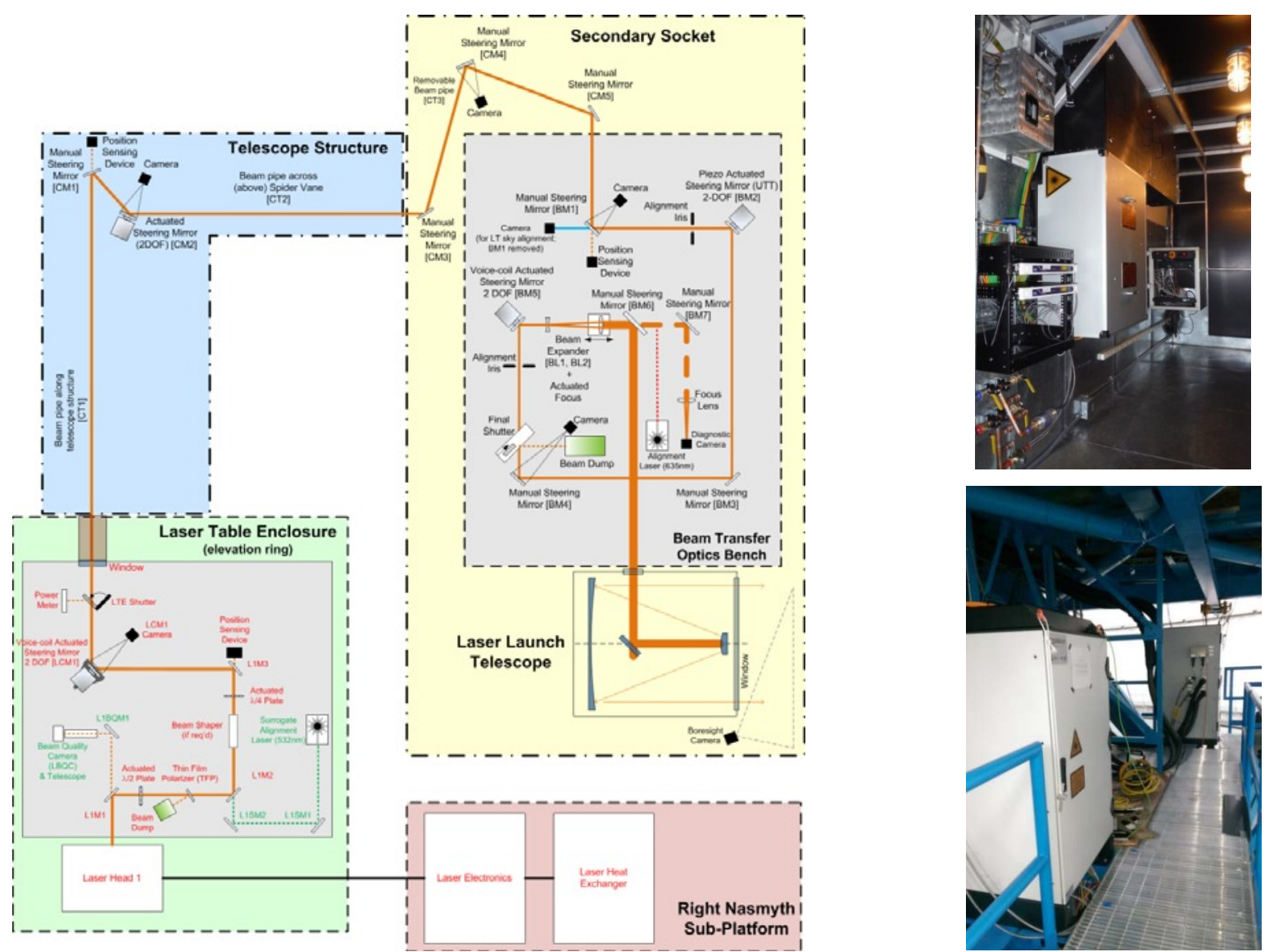

Figure 1: Schematic diagram of the K2 NGL system with the CLS (left); the laser table enclosure and laser head on the telescope's elevation ring (top right); the laser platform housing the laser electronics cabinet and laser heat exchanger (bottom right). 
The functional layout of the NGL and the CLS is shown in Figure 1. The blue and yellow areas are the components that were installed as part of the CLS. The areas in red and green are installed as part of the NGL system. The laser itself is divided into two physical areas. The laser electronics cabinet (EC) and the heat exchanger (HEX) are located on a platform with a fixed gravity vector while the laser head (LH) is located on the telescope's elevation ring. This configuration is known as the TOPTICA/MPBC's remote pumping format. The laser components are connected with 30 $\mathrm{m}$ of fibers and cabling between the LH and EC. TOPTICA/MPBC also supports a laser version known as local pumping for shorter distances between the EC and LH. The primary difference is the need for higher power levels in the diode pumps to overcome fiber losses and filtering to reduce stimulated Brillouin scattering (SBS). In addition to the LH at the elevation ring, an optical bench known as the Laser Table Enclosure (LTE) provides the necessary functionalities to steer the beam into the CLS.

\subsection{Laser Platform}

Figure 2 shows the physical platform and the model of the enclosure on the elevation ring where the LTE and LH are located. The platform houses a single EC and the HEX exchanger. Observatory power and coolant are provided at the platform to support the laser system. Figure 2 also shows a rendering of the platform and enclosure with two additional laser systems. The HEX is capable of supporting three lasers and the current infrastructure has been implemented to support the future lasers. The elevation ring enclosure mechanical components are designed for two additional LHs to be mounted and the LTE is sized to combine and steer three laser beams into the CLS beam train. The design and implementation of these upgrades are incremental steps toward the NGAO system using a seven laser beacon asterism.
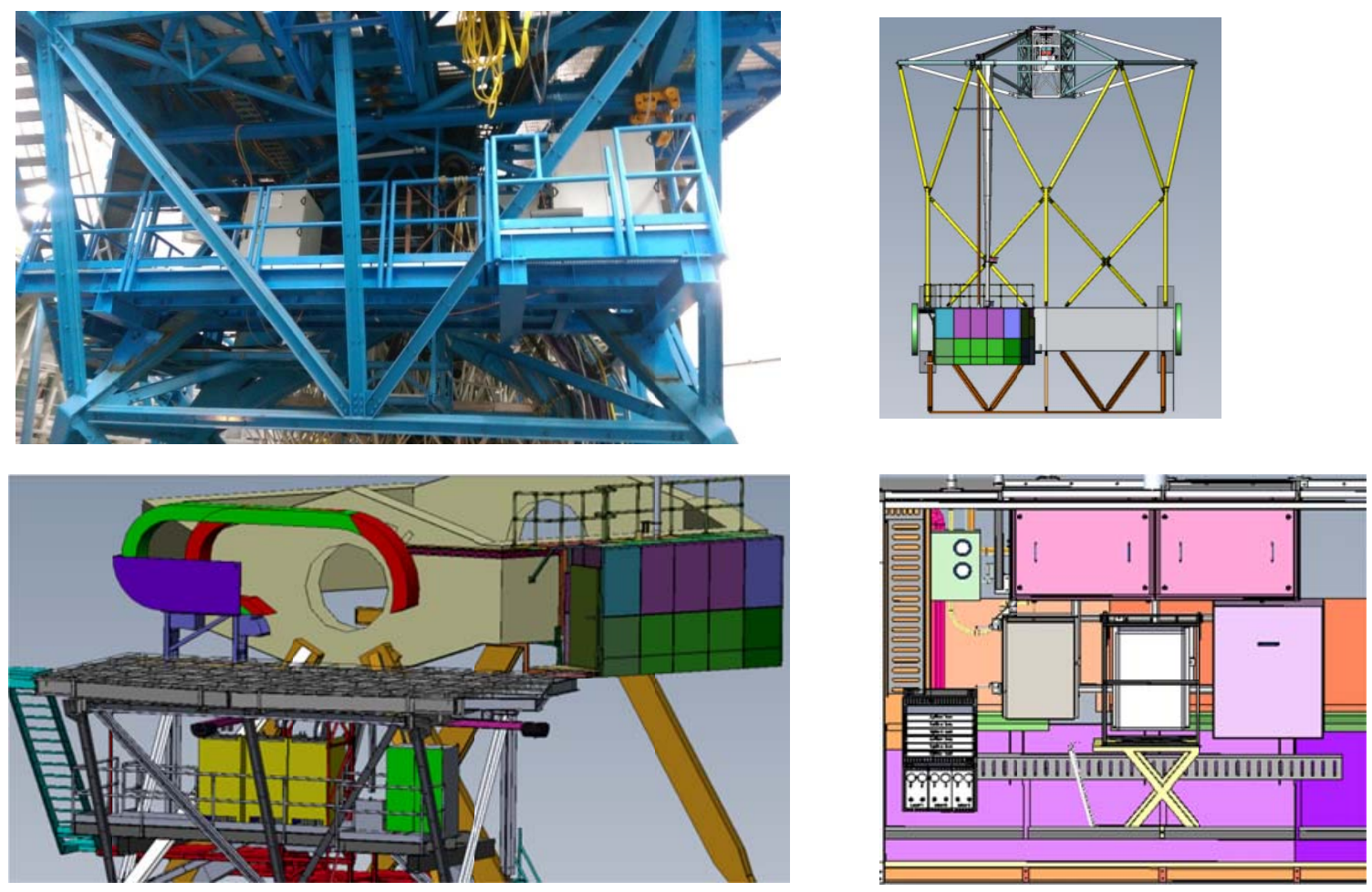

Figure 2: Laser platform under the telescope's Nasmyth platform with a single laser system and the heat exchanger (top left); rendering of the laser platform with 3 TOPTICA/MPBC lasers with heat exchanger (bottom left); elevation ring enclosure with launch tubes (top right). Rendering of elevation ring enclosure with three laser heads (bottom right)

\subsection{Laser Table Enclosure}

The LTE's primary function is to steer the beam from the laser into the CLS. Pointing and centering control is necessary to address flexure and vibration on the telescope as it moves in elevation. The LTE (Figure 3) is located on the telescope's elevation ring which is extremely stiff. The first mirror on the beam train is $13 \mathrm{~m}$ away on the telescope's top 
ring which flexes in both azimuth and elevation. Without closed loop control, the beam can move as much as $19 \mathrm{~mm}$ in the beam train at various mirrors (Chin et al. [6]). The LTE also provides one of three safety shutter controls in the beam train. The amount of power exiting the LTE is controlled via a $1 / 2$ waveplate (WP) that rotates the linearly polarized light and a thin film linear polarizer (TFP) that reflects part of the light into a beam dump. The laser itself has power adjustment with a range of 16 to $22 \mathrm{~W}$; the WP/TFP combination allows low power $(\geq 1 \mathrm{~W})$ levels for alignment purposes. Unused laser power from the shutter mirrors or during alignment is removed via glycol cooled beam dumps. A quarter WP converts the laser's linear polarization to circular polarization to maximize sodium return.

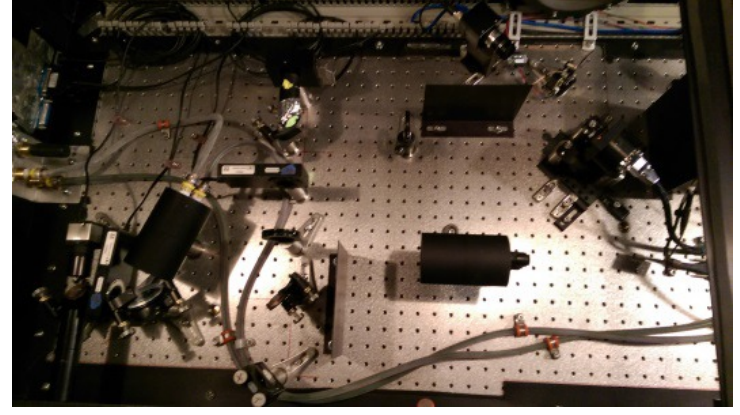

Figure 3: Laser Table Enclosure components.

Diagnostics in the LTE are used to monitor the beam's performance and wavelength. Three mirrors, with position diagnostics, ensure the laser beam's pointing and centering alignment into the beam train. The TOPTICA/MPBC LH has an option to include output steering mirrors to support non-real time alignment purposes; these motors were measured to have a resolution of 0.0125 to $0.013 \mu \mathrm{rad}$ per step. A Shack-Hartmann camera on the LTE can be used to examine the laser's beam quality. In addition to the laser's internal wave meter, a sodium cell independently verifies the wavelength without the need to go on sky. The LTE also includes a surrogate laser for basic alignment checks if the sodium laser is not available; this laser hasn't been required. The LTE and its mounting frame's physical dimensions are sufficiently sized to support two additional $\mathrm{LH}$ in the future.

\subsection{Laser Safety and Safety System}

The TOPTICA laser operates as a class IV laser system and complies with the IEC 60825-1 Part 5 Manufacturer's Checklist. The requirement for a laser safety system (LSS) to operate with the TOPTICA laser is guided by ANSI standards Z136.1 Safe Use of Laser for Indoors and Z136.6 Safe Use of Laser for Outdoors. This guidance ensures personnel and equipment safety via engineering (interlocks) and procedural controls. The previous LSS was upgraded for obsolescence as part of the NGL project with an Allen Bradley SLC500 Programmable Logic Controller (PLC). To ensure there is no dependency on any network, the LSS runs independently with a hardware interface directly to the laser. The laser itself has an internal PLC to maintain internal safety. The NGL PLC monitors and controls the critical functionalities such as safety shutters in the beam train to prevent unintended radiation exposure. It also serves to gather the necessary permissives that permit the propagation of the beam onto the mesosphere. These permissives include the satellite and aircraft avoidance, beam avoidance of other telescope's observing paths, telescope and dome positions, beam pointing accuracies, and the general health of the laser and associated subsystems. Loss of communication heartbeats among systems signals a lack of control, resulting in shutter closures.

\subsection{Laser Software and Interface}

The software architecture for the laser is shown in Figure 4. A single Linux server controls and communicates with the laser, safety system, CLS beam train motion electronics, environmental monitoring, and other subsystems on the observatory network. The server runs Red Hat Enterprise Edition 7 in the observatory's EPICS software environment. This allows all other clients such as the AO system and instruments to control and access laser status via keywords. A graphical user interface (GUI) called the CLS Sequencer (Figure 5) provides user control of the beam train as well as the laser. This interface depicts a physical layout of the laser and components within the beam train. Real time operating parameters and status are provided over each component icon. Operational controls of the laser modes and beam train components can all be performed with this single GUI. In addition to the four operational modes of the laser (STANDBY, READY, ON, OBSERVATION), advanced laser parameters such as wavelength, power, and repumping can be accessed via pull down menu/screens. Critical laser parameters and control are provided via the laser service software on a laptop. Inter subsystem communications status are provided via colored LEDs to inform the user of faults. A separate visual and audio alarm system informs the user of faults and provides guidance for recovery. The Linux server also serves as a repository of data logging by the EPICS system as well as logs from the TOPTICA service laptop. Laser parameters can be logged with the EPICS system using channel monitoring via TCPIP. 


\subsection{Laser Service Software Interface}

The TOPTICA/MPBC laser is delivered with a Windows laptop running TOPTICA service software (TSS, Figure 6) to communicate with the laser directly via TCPIP. For normal night time operations, the laptop is not used and is not required to operate with the laser. All controls are done by the telescope operator using the CLS Sequencer. The TSS has extensive controls and viewing of internal laser parameters. Each laser subsystem has a panel to command operational set points and view status. To ensure safety, the software has three levels of controls, NORMAL, MAINTENANCE, and SERVICE. Each level provides the user with control features which can bypass interlocks. Only trained technicians or engineers are permitted to operate the TSS. To support troubleshooting, the software provides constant logging of parameters and history. Status and transition history are located on two panes in the software. To minimize memory usage, the logs are transfer to the Linux server daily. The TSS also includes non TOPTICA software to examine internal system components such as the Siemens PLC and MPBC's diode pump system. Together with plotting and logging, the user is easily able to troubleshoot and identify problems.

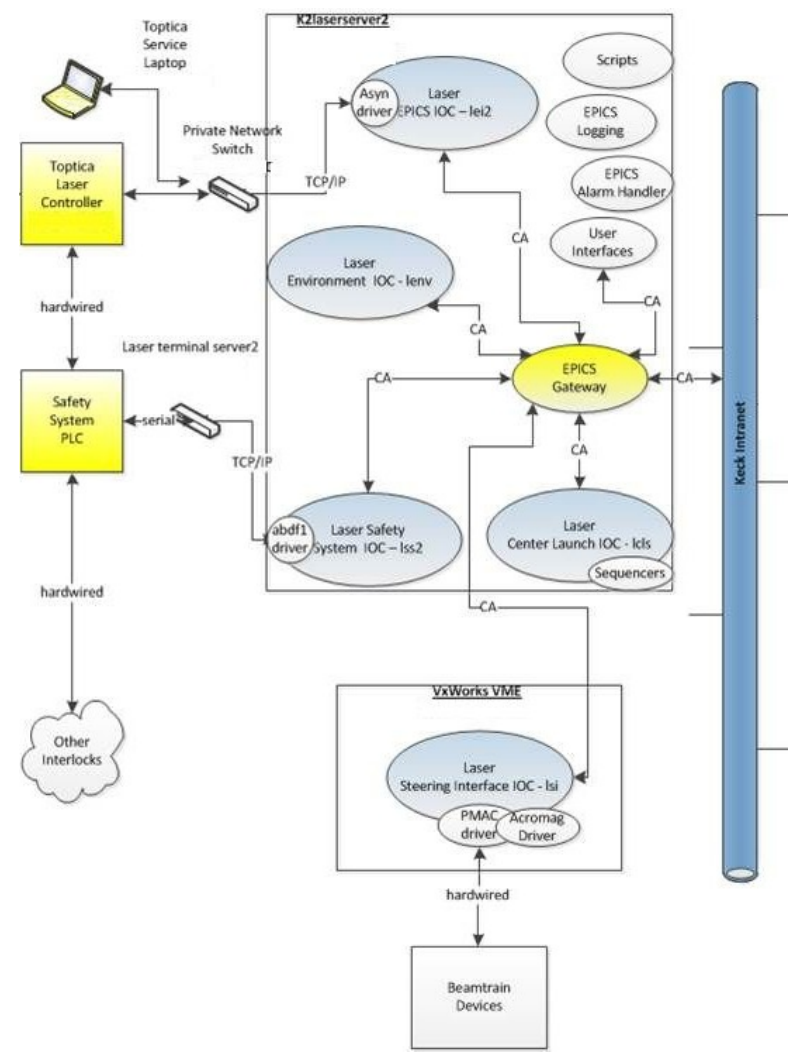

Figure 4: Laser Software Architecture

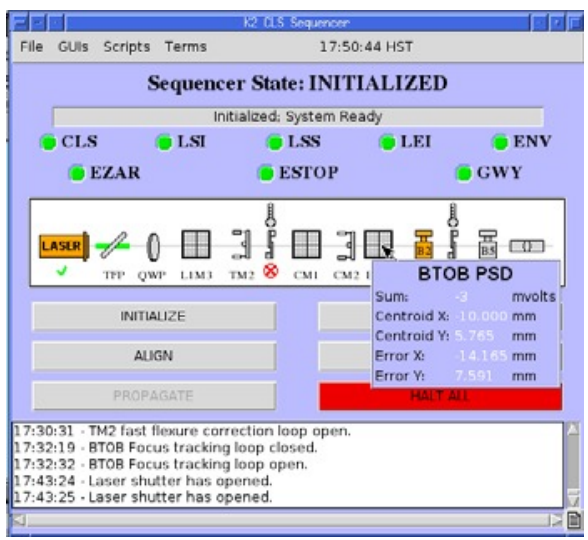

Figure 5: Keck CLS Sequencer user interface.

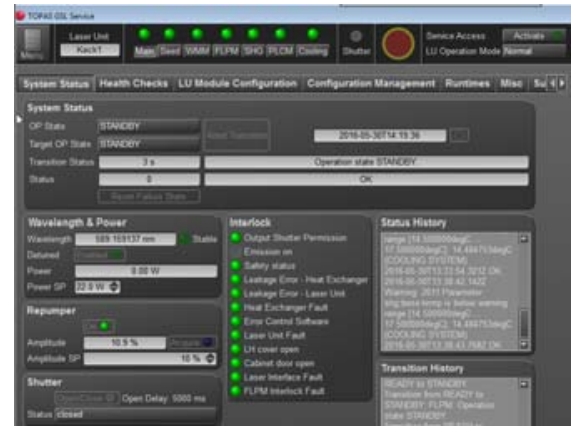

Figure 6: TOPTICA laser service software interface.

\section{LASER PERFORMANCE}

The laser's performance and functional acceptance testing were completed in four phases. The first phase of testing was conducted at the factory in Germany in September of 2014. All specifications and requirements were verified at the factory. The second phase was testing of the laser at the Keck headquarters in an environmentally controlled laboratory to more closely simulate the summit temperature environment $\left(0{ }^{\circ} \mathrm{C}\right)$; due to high humidity at the Keck headquarters, the system was operated at $9.2{ }^{\circ} \mathrm{C}$ to prevent condensation. The laser was then transferred onto the Keck II telescope for testing in the dome operating environment; humidity is normally $<30 \%$ at the summit of Maunakea. In this third phase, the HEX, EC, and the LH were all located on the newly installed laser platform. The LH/LTE which replaces the DL amplifier optical bench on the elevation ring was not installed into their final configuration since the DL had not yet been decommissioned. The laser platform testing was completed in September, 2015. In the final phase, the dye laser was removed and replaced with the LH/LTE for the final commissioning. The risks at this point were minimal as the 
laser had already been tested in the dome environment. The data presented in the next sections are from all phases when it was practical to measure.

\subsection{Laser Output Power and Stability}

The laser output power is set at $22 \mathrm{~W}$ with $90 \%$ of the power in the $\mathrm{D}_{2 \mathrm{a}}$ line and $10 \%$ of the power in the $\mathrm{D}_{2 \mathrm{~b}}$ repumper lines. This power is divided among the $\mathrm{D}_{2 \mathrm{a}}, \mathrm{D}_{2 \mathrm{~b}}$ red, and $\mathrm{D}_{2 \mathrm{~b}}$ blue lines. There is asymmetry in the $\mathrm{D}_{2 \mathrm{~b}}$ power generation with $1.1 \mathrm{~W}$ being lost in the $\mathrm{D}_{2 \mathrm{~b}}$ red sideband; $1.9 \mathrm{~W}$ of $\mathrm{D}_{2 \mathrm{~b}}$ blue sideband power is generated along with $19 \mathrm{~W}$ of $\mathrm{D}_{2 \mathrm{a}}$ power. Figure 7 shows data collected on May 2, 2016 UT taken by the laser. The initial power ramp is due to temperature stabilization of the LH after being turned on. It takes just under an hour for the laser head temperature to stabilize after power is applied to the laser cavity and RFA. The output power is stable except for periods of detuning and retuning sequences when the wavelength is offset by $5 \mathrm{GHz}$. During detuning and retuning transitions, both the wavelength and the cavity are unstable. The measurements for these periods are removed from the data. It is worthwhile noting that the power tends to change by a maximum of $0.1 \mathrm{~W}$ after a detuning sequence; these shows up as steps in the power in Figure 7. Telescope gravity vector changes do not seem to affect the laser output power as much as the detuning sequences.

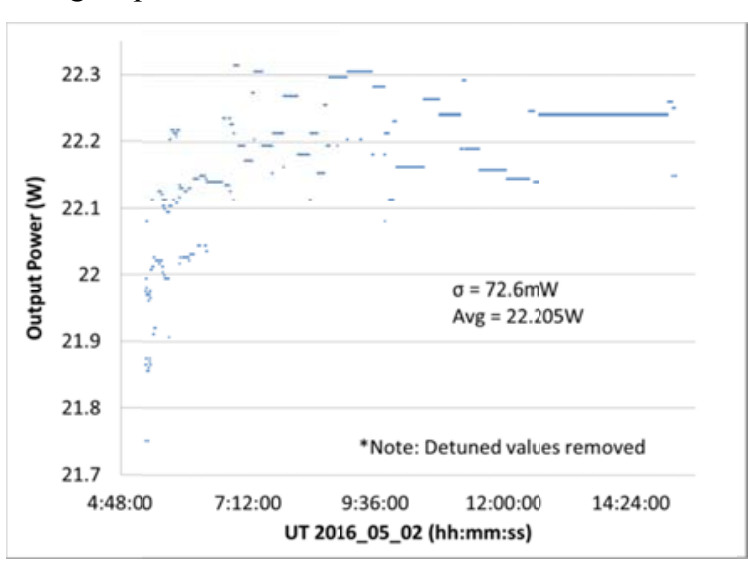

Figure 7: Laser output power measured by the laser. Detuning sequences have been removed from the data due to its power instability during the detuning periods and transitions.

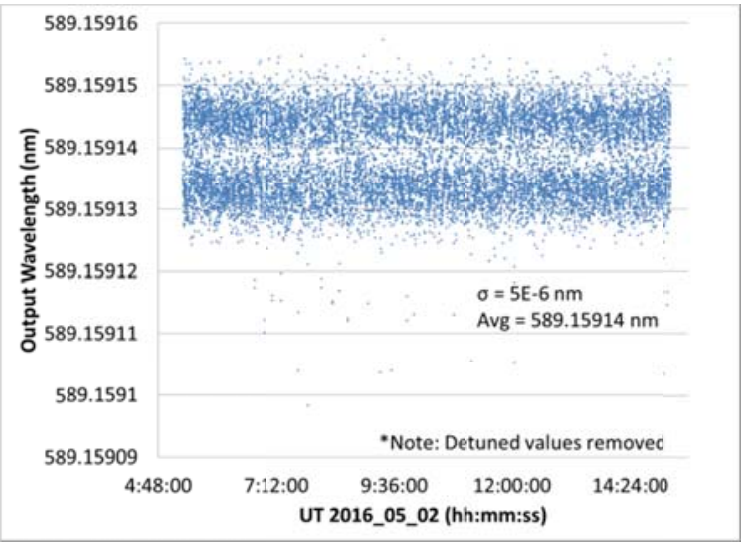

Figure 8: Laser output wavelength measured by the laser. Detuning sequences have been removed from the data due to its wavelength instability during the detuning periods and transitions.

\subsection{Laser Output Wavelength and Stability}

The wavelength for the laser is controlled by a seed laser set at $1178.318278 \mathrm{~nm}$. This value can be arbitrary set within a 6 picometer range with a resolution of $0.001 \mathrm{pm}$; however, variations in the sodium return measured by the WFS are seen only at the $0.1 \mathrm{pm}$ level. The sodium $\mathrm{D}_{2 \mathrm{a}}$ output wavelength is the result of second harmonic generation from this seed wavelength. Figure 8 shows the data from the same observing night that the power was measured. Similarly, the detuning and retuning periods when wavelength is shifted is removed; several seconds of data after the retuning sequence is also removed as the wavelength is unstable during the retuning transition. The plot shows the wavelength is transitioning between two states at the $0.01 \mathrm{pm}$ level as measured by the laser's internal wave meter, well within the $\pm 5 \mathrm{pm}$ resolution specification. The sigma is within the $\pm 40 \mathrm{MHz}$ stability requirement of the laser.

\subsection{Laser Output Beam Quality}

The laser's beam quality was measured with a Thorlabs Shack-Hartmann camera behind the first fold mirror in the LTE (Figure 9). The beam diameter is $2.450 \times 2.426 \mathrm{~mm}(\mathrm{x}, \mathrm{y})$, meeting the beam size and ellipticity

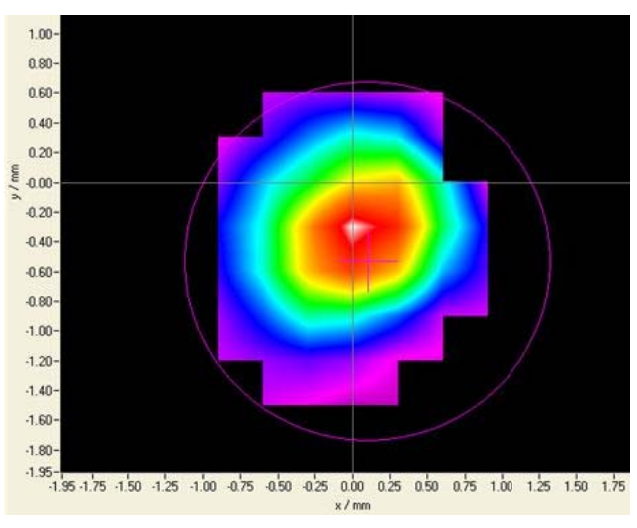

Figure 9: Beam quality measurement of the laser beam at the LTE. 
requirement. The wavefront error is measured to be $29 \mathrm{~nm}$ rms with the dominant error term being defocus; this is less than half of the specified wavefront error requirement. Although this is the largest term, the beam remains well collimated in the beam train at $20 \mathrm{~m}$ from the laser source. A pair of lenses is used just before the launch telescope to expand the beam and account for any focus adjustments to minimize the laser spot size on the sky. A model is also applied to the lens pair to account for launch telescope changes due to elevation angle and temperature.

\subsection{Laser Power Usage}

One key advantages of the NGL is its efficiency and ease of operation. As compared to dye laser operation, the NGL has significantly reduced human and infrastructure resources needs. The TOPTICA laser (North American version) uses 3phase $208 \mathrm{VAC}$ as its primary input voltage. An uninterruptable power supply is used to minimize power fluctuations and disruptions. Unlike the dye laser which consumed $50 \mathrm{~kW}$ of power and required an additional $30 \mathrm{~kW}$ to remove the heat from the facility, the NGL consumes significantly less power and requires much less heat removal. Table 1 shows the TOPTICA laser power usage in its operating states excluding the HEX. The power used by the laser is less than a typical observatory instrument. HEX power consumption is highly dependent on the pump speed necessary to provide the required flow to the EC and LH. The HEX, which includes the pump, consumes $0.39 \mathrm{kVA}$. This power does not include the $2 \mathrm{~kW}$ heaters in the HEX and LH to maintain the coolant and LH temperatures when the laser is powered OFF. The required power to drive the diodes can also increase by a factor of 2 during the lifetime of the diodes.

\begin{tabular}{|l|c|c|c|}
\hline \multirow{2}{*}{ Mode } & \multicolumn{2}{|c|}{ Power } & \multirow{2}{*}{ Power Factor } \\
\cline { 2 - 4 } & kW & kVA & \\
\hline Standby & 0.19 & 0.29 & 0.64 \\
\hline Ready & 0.19 & 0.29 & 0.64 \\
\hline On & 0.54 & 0.6 & 0.9 \\
\hline Observation & 0.55 & 0.61 & 0.89 \\
\hline
\end{tabular}

Table 1: Laser power consumption based on the various states of the laser

\begin{tabular}{|l|c|}
\hline Transitions & Time (sec) \\
\hline Standby to Ready & 3 \\
\hline Ready to On & 195 \\
\hline $\begin{array}{l}\text { Ready to On with } \\
\text { wavelength calibration }\end{array}$ & 2895 \\
\hline On to Observation & 9 \\
\hline
\end{tabular}

Table 2: Laser transition times. There is a $5 \mathrm{sec}$ preset delay for the shutter to open in the observation mode.

\subsection{Laser Control and Transition Times}

The TOPTICA/MPBC laser is much simpler to operate than the previous dye laser. The laser has four states/modes of operations. The system is in OFF mode when there is no power to the EC. Heaters in the LH and HEX are used to maintain temperatures even when there is no power to the EC. Once power is applied to the EC, the laser goes to the STBY mode. It takes about 5 minutes for controllers within the laser to connect with the observatory interface awaiting further commands. Once the connection is made, the laser is commanded to the READY state. In this transition, the laser closes internal loops prior to turning ON the diodes. If necessary, the seed laser is calibrated at this time. A 240 hour timer is used to determine wavelength calibration intervals. Once in the READY state, the laser diodes are enabled transitioning to the ON state. In the final transition to the OBSERVATION (OBS) state, the laser output shutter is opened. The laser can be commanded from OFF to OBS in a single command without user intervention as long as temperatures and power levels within the laser are in acceptable ranges. The time to transition each state is shown in Table 2. The transition timing assumes the laser has been in the STANDBY state, allowing it to thermalize from the day prior. All of the controls and system monitoring can be done remotely by the laser technician.

\section{LASER ON-SKY PERFORMANCE}

The NGL system first light was on December 1, 2015. On-sky engineering and commissioning followed with LGSAO science operations with the new laser returning on April 15, 2016. During this period, the laser was on sky for 7 nights with 1.5 of the 7 nights lost due to weather. The test data below are from the on-sky testing and subsequent science nights. The performance data is also compared to the Keck II dye laser center and side launch and to the Keck I solid state laser center launch.

\subsection{Sodium Return Brightness}

The TOPTICA laser format was designed to optimize the photon return from the sodium layer. The laser's power, continuous wave format, narrow line width, optical pumping of the $\mathrm{D}_{2 \mathrm{~b}}$ sodium line and linear polarization are all factors in improving the sodium return. The return is also dependent on the laser pointing direction with respect to the earth's 
magnetic field (Holzlöhner et al. [12]). Figure 10 shows the NGL return as compared to the dye laser in the SLS and CLS configurations. The NGL system operates only in the CLS configuration. The plot shows normalized frequency of occurrence versus the LGS equivalent R magnitude. The median dye laser brightness in the SLS configuration for high elevations $\left(70-87^{\circ}\right.$ ), based on 2728 measurements taken during Apr. 2013 through Jun. 2015, is $\mathrm{R}=10.2 \pm 0.4$. The median dye laser brightness in the CLS configuration for high elevations $\left(70-87^{\circ}\right)$, based on 750 measurements taken during Apr. 2014 through Oct. 2015, is $\mathrm{R}=10.7 \pm 0.4$. The 0.5 magnitude photon return reduction in going from dye laser SLS to CLS is due to the additional throughput losses in the CLS beam train; the CLS added 14 fold mirrors, two waveplate, a thin film power polarizer, 4 lenses, and 2 windows to the beam train. The median NGL brightness in the CLS configuration for a narrower elevation range ( $75-87^{\circ}$; since the NGL return depends heavily on pointing direction), based on 1630 data points taken on Jan. 5, 2016 UT (after optimizing the launch optics alignment, focus, laser wavelength and polarization), is $R=7.5 \pm 0.2$.

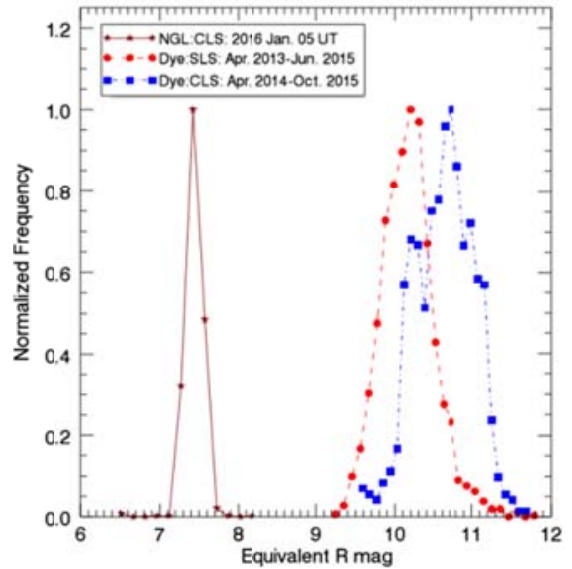

Figure 10: Normalized histogram of the sodium return in R magnitudes of the NGL with CLS, DL with CLS, and the DL with SLS.

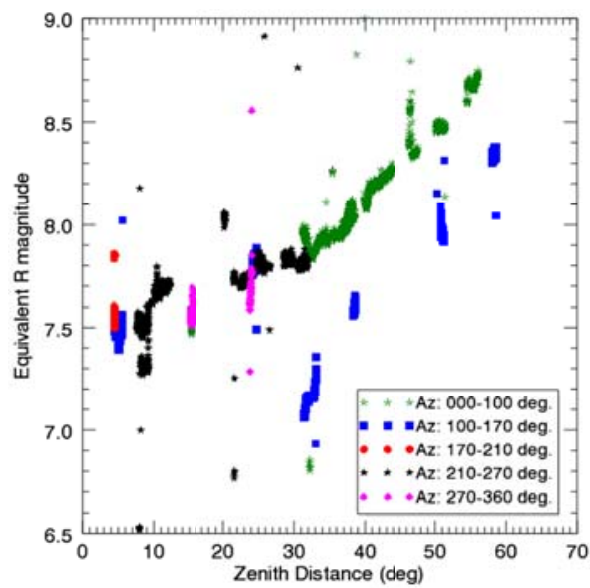

Figure 11: NGL sodium return in R magnitudes versus pointing direction (January 05, 2016 UT)

Figure 11 shows the NGL equivalent R magnitude dependency on laser pointing direction. The NGL brightness measurements taken at all elevations are plotted against zenith distance for different azimuth ranges. The elevation and azimuth dependent laser return is apparent from this figure.

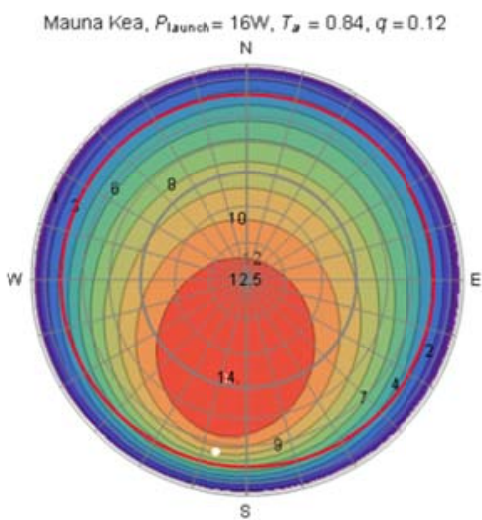

Figure 12: Expected sodium returns from Bloch models for Maunakea site with a TOPTICA laser; units of $\mathrm{Mph} / \mathrm{m}^{2} / \mathrm{sec}$ (Holzlöhner et al. [12]).

\begin{tabular}{|l|c|}
\hline Parameter & Value \\
\hline $\begin{array}{l}\text { Laser power launched; includes the power in } \\
\text { the D2b blue sideband }\end{array}$ & $13.53 \mathrm{~W}$ \\
\hline $\begin{array}{l}\text { Polarization; use of } 1 / 4 \text { waveplate to convert } \\
\text { laser's linear polarization }\end{array}$ & Circular \\
\hline $\begin{array}{l}\text { D2b repumping power fraction (Optimum } \\
\text { value from model 12\%) }\end{array}$ & $10 \%$ \\
\hline $\begin{array}{l}\text { Repumping frequency offset (Optimum value } \\
\text { from model 1.7178 GHz) }\end{array}$ & $1.713 \mathrm{GHz}$ \\
\hline Atmospheric Transmission & 0.84 \\
\hline Beam Transport Throughput & 0.871 \\
\hline Launch Telescope Throughput & 0.924 \\
\hline Na Column Density & $3.75 \times 10^{13}$ atoms $/ \mathrm{m}^{2}$ \\
\hline Sodium Altitude & $92 \mathrm{~km}$ \\
\hline Propagating Altitude & $4.2 \mathrm{~km}$ \\
\hline Seeing & $0.5 \mathrm{to} 0.7^{\mathrm{\prime}}$ \\
\hline 1/e beam diameter & $0.36 \mathrm{~m}$ \\
\hline Laser Power & $20 \mathrm{~W}$ \\
\hline Spot Size & $1.6^{\prime \prime}$ \\
\hline
\end{tabular}

Table 3: Parameters for sodium return calculations.

Figure 12 is taken from Holzlöhner and predicts a return of $14 \mathrm{Mph} / \mathrm{m}^{2} / \mathrm{sec}$ at the optimal pointing direction with a $16 \mathrm{~W}$ TOPTICA laser launched for the Maunakea site with atmospheric transmission of 0.84 . Using the dye laser efficiency 
measurement of $1.15 \mathrm{Mph} / \mathrm{m}^{2} / \mathrm{sec}$ in 2005 when the $\mathrm{K} 2$ dye laser was first commissioned ([LeMignant et al. [9] \& Neyman [10]), scaling to $1.01 \mathrm{Mph} / \mathrm{m}^{2} / \mathrm{sec}$ for the lower laser operating power, and the R magnitude gain from Figure 10 , the TOPTICA laser return is scaled to $12.13 \mathrm{Mph} / \mathrm{m}^{2} / \mathrm{sec}$ for elevation angles of $75^{\circ}$ and higher. This translate to $0.90 \mathrm{Mph} / \mathrm{m}^{2} / \mathrm{W} / \mathrm{sec}$ for the TOPTICA laser as compared to Holzlöhner model of a $1.04 \mathrm{Mph} / \mathrm{m}^{2} / \mathrm{W} / \mathrm{sec}$. Aside from the model, there are several factors not included in the measured value. First, the repumping frequency offset for the $\mathrm{D} 2_{\mathrm{b}}$ is not optimized. Based on measurements by Bonaccini et al. [11], an increase of $6 \%$ in return can be gained by optimizing the $\mathrm{D} 2$ b frequency from 1.713 to $1.7178 \mathrm{GHz}$. The Keck measurements are collected with the laser pointed at high elevation angles with various azimuth angles, which is not at the optimal location of $65^{\circ}$ elevation angle pointing south. Along with spot size, these factors may account for the lower than expected efficiency in the model.

\subsection{Sodium Coupling Efficiency}

The sodium coupling efficiency $S_{c e}$ of the NGL is examined as compared to the Keck II SLS dye laser. The SLS dye laser has a return flux of $1.01 \mathrm{Mph} / \mathrm{m}^{2} / \mathrm{s}$ and is scaled to $12.13 \mathrm{Mph} / \mathrm{m}^{2} / \mathrm{s}$ with the improved NGL return. Using the parameters and conditions in Table 3 and the $S_{c e}$ equation from Holzlöhner et al. [13], where $F_{m}$ is the return flux on the receiver, $\Phi$ is photon flux on the detector, $L$ is the distance to the sodium beacon, $P(T a)^{X}$ is the laser power to the mesosphere, and $C_{n a}$ is the sodium column density, the $S_{c e}$ is calculated to be 261 photons $/ \mathrm{s} / \mathrm{sr} /\left(\mathrm{W} / \mathrm{m}^{2}\right) /$ atom. The calculation assumes an airmass of 1 . Since no independent measurement of the sodium column density was taken, a median estimate of the density over the Hawaiian Islands from January to April was used from Roberts et al. [14]. This matches well with the Bloch modelling (Mathematica Atomic Package together with the Bloch Equations Mathematica code)for a 1.6" FWHM sodium laser spot at $10.35 \mathrm{~W} / \mathrm{m}^{2}$ in the mesosphere within $4 \%$ for a circular polarized beam (Figure 13).

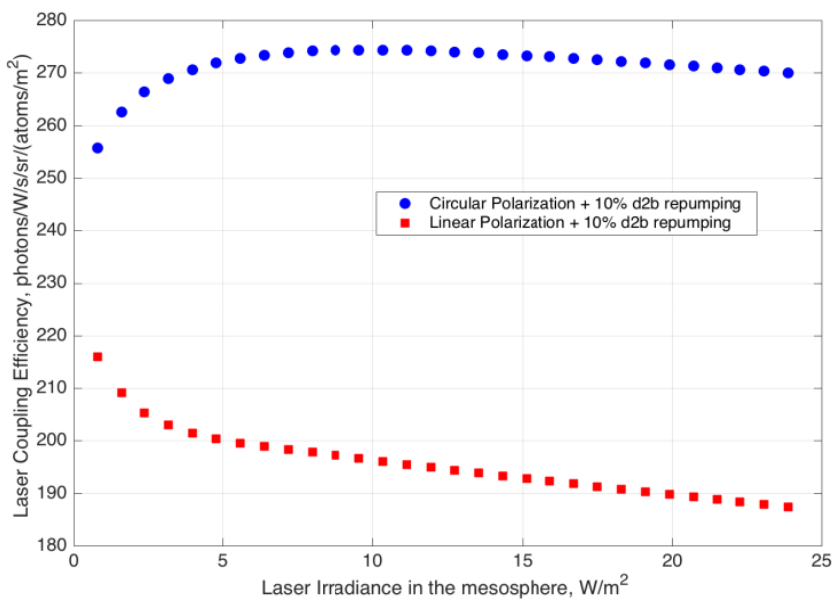

$$
s_{c e}=\frac{F_{m}}{P\left(T_{a}\right)^{X}}=\frac{\Phi L^{2}}{P\left(T_{a}\right)^{2 X} C_{\mathrm{Na}} X}
$$

Figure 13: Sodium coupling efficiency formula (left). Laser coupling efficiency at the Keck site based on laser irradiance and spot size of 1.6 arc sec. WMKO irradiance $=10.35 \mathrm{~W} / \mathrm{m}^{2}$.

\subsection{Rayleigh Scattering}
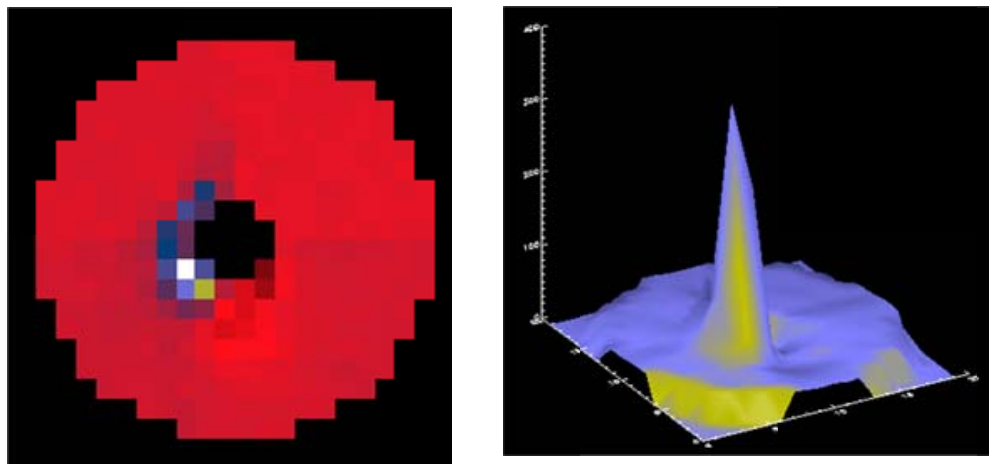

Figure 14: Wavefront sensor image of the Rayleigh return at $30^{\circ}$ elevation and on Jan 22, 2016 HST with cirrus conditions (left); single sub-aperture having an R magnitude of 10.3 as compared to the LGS magnitude of 8.5 . Rayleigh is off center likely due to a pointing misalignment out of the launch telescope (right).
The relative contribution of Rayleigh scattering in the atmosphere has been reduced with the significant increase in sodium return with the NGL. At high elevations, the telescope's secondary mirror socket and module block the Rayleigh scattering. At low elevations, the Rayleigh scattering occurs at larger distances from the telescope, and high altitude scattering may not be blocked by the secondary. Rayleigh scattered light on the wavefront sensor is seen mostly at low elevations or on nights with high cirrus. Using the TOPTICA detuning function, the laser's frequency is offset by $5 \mathrm{GHz}$, removing the LGS and leaving the Rayleigh background to be subtracted. On the night of January 5, 2016 UT the equivalent Rayleigh R 
magnitude on the wavefront sensor at $40^{\circ}$ elevation angle was 13 compared to the LGS magnitude of 7.8. Figure 14 shows the Rayleigh at $30^{\circ}$ elevation angle when detuned on a cirrus night. The return brightness of one sub-aperture has an R magnitude of $\sim 10.3$ (compared to the 8.5 magnitude LGS); the average background is 25 counts compared to the Rayleigh peak of 300 counts. Rayleigh scattering and sky background are automatically subtracted by the wavefront sensor during acquisition using the detuning function.

\subsection{Repumping Gain}

Figure 15 shows the laser return on the wavefront sensor with and without the repumper at a $10 \%$ fraction of the output power. The laser polarization was optimized for circular polarization with a return spot size of $1.6^{\prime \prime}$ at zenith. An increase of $61 \%$ in return can be seen with the repumper on at the laser's maximum $22 \mathrm{~W}$ setting. This data matches well with the sodium return model for a 1.6" FWHM laser spot on-sky as shown in Figure 16. Based on this spot size, the gain from the repumper ON vs OFF is a factor of 1.6. Figure 15 also shows the return continuing to increase (i.e. no saturation) as the laser output power is increased from 16 to $22 \mathrm{~W}$. The laser also has the ability to change the repumping percentage from 0 to $10 \% ; 12 \%$ have been shown to be the maximum. Figure 17 shows the return intensity as the repumping is ramped from 5 to $10 \%(0 \%$ equates to the repumper in the OFF setting) and nearing saturation with $10 \%$ repumping.

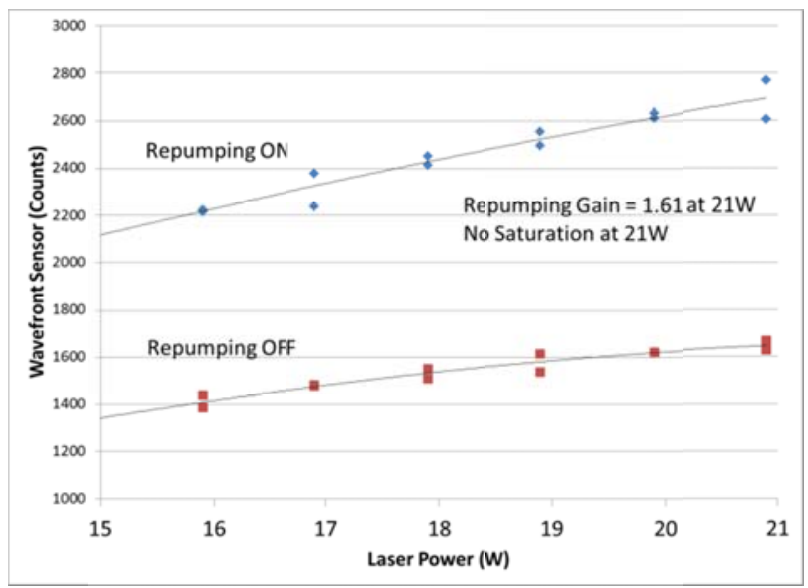

Figure 15: Laser return as measured on the wavefront senor at various laser power settings with and without the D2b repumping ON (10\%) and with circular polarization. (Dec. 22, 2016 05:07UT; telescope at zenith).

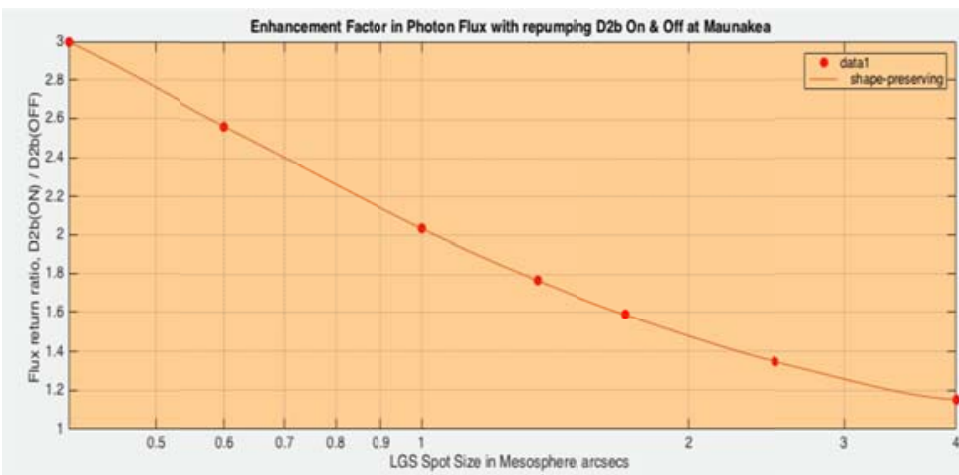

Figure 16: Flux return model of ratio with and without repumper ON vs laser FWHM spot size.

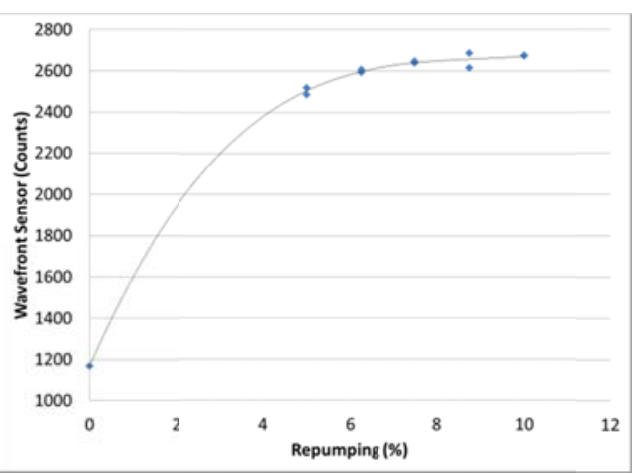

Figure 17: Laser return as measured on the wavefront sensor at various laser repumper \% settings with circular polarization. (Dec. 22, 2016 05:17 UT. Telescope at zenith position)

\subsection{Polarization Effect}

The impact of the laser beam's polarization can be seen in Figure 18. The laser output is linear polarized to a factor $>220$ by measurement of the extinction ratio at the factory. A quarter waveplate is located on the LTE optical bench and is rotated to determine the optimal return on the wavefront sensor. Maximum return should be achieved with circular polarized laser light exciting the atomic energy transitions of maximum absorption. Since there isn't an easy method to measure the polarization of the $180 \mathrm{~mm}$ diameter output beam at the launch telescope, the maximum and minimum wavefront sensor values are used to provide an estimate of the gain from circular to linear polarized laser light. The nonsymmetry is likely due to the 14 fold mirrors at different incidence \& compound angles between the quarter waveplate and the output of the launch telescope as the waveplate is changed. The gain is measured to be $39.9 \%$ which matches well with the Bloch model for a laser irradiance of $10.35 \mathrm{~W} / \mathrm{m}^{2}$ (Figure 19). Similar gains (30-40\%) using a TOPTICA like laser were completed by ESO at Tenerife by Bonaccini et al. [11]. 


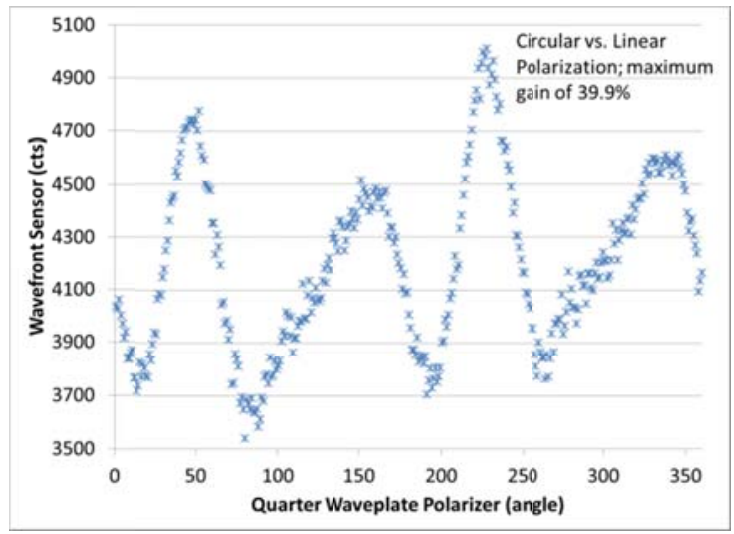

Figure 18: Laser return as measured on the wavefront sensor as the laser beam is varied between circular and linear polarized light. (Dec. 18, 2016 09:59 UT; telescope at zenith position).

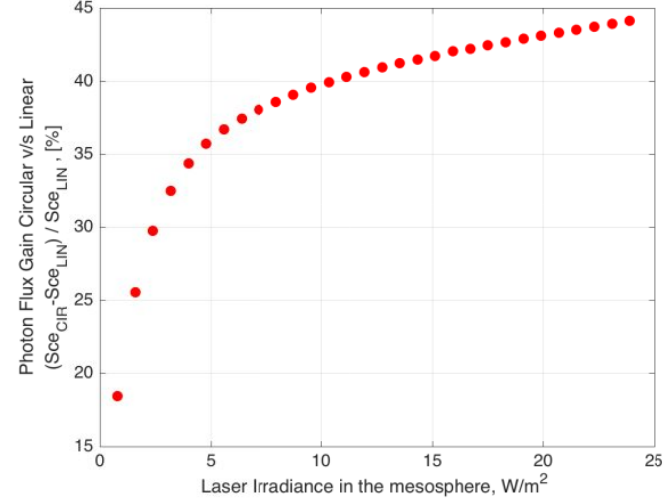

Figure 19: Laser model gain between circular and linear polarization. Laser irradiance is $10.35 \mathrm{~W} / \mathrm{m}^{2}$.

\subsection{Laser Spot Size and Elongation}

The LGS spot size is measured with an acquisition camera on the AO bench. The camera has a 120" field of view with 0.134 arc sec pixel size. This camera is used at the start of an observing night to ensure the laser beam is optimized in focus and position on the wavefront sensor with the telescope at zenith. The laser pointing model is based on this initial position. Focus of the output beam is adjusted via a lens pair located before the launch telescope. A model is used to compensate for temperature and elevation. The laser focus is generally stable once it is set at the start of the night. Figure 20 (left) shows the measured LGS spot size at zenith for the center launch TOPTICA laser, center launch dye laser, and side launch dye laser as a function of seeing. Typical TOPTICA laser spot sizes are 1.5"x1.6" Overall, the CLS spot size is $24 \%$ smaller than that of the SLS. For comparison, the Keck I CLS spot size is plotted in Figure 20 (right).

An examination of the spot size is analyzed in Ragland et al. [15] on the measured spot size as compared to the theoretical model. The sodium-thickness-free spot size is estimated from the FWHM of the unstacked images in the azimuthal direction with a value of 1.06" (Figure 22). Combining the broadening due to finite sodium layer thickness, atmospheric seeing in double pass, and the finite beam size, an image of $1.2 \pm 0.1$ " should be achieved. The difference in the achieved vs. this predicted value is likely due to launch optics aberrations, focus error, laser collimation, and beam divergence.
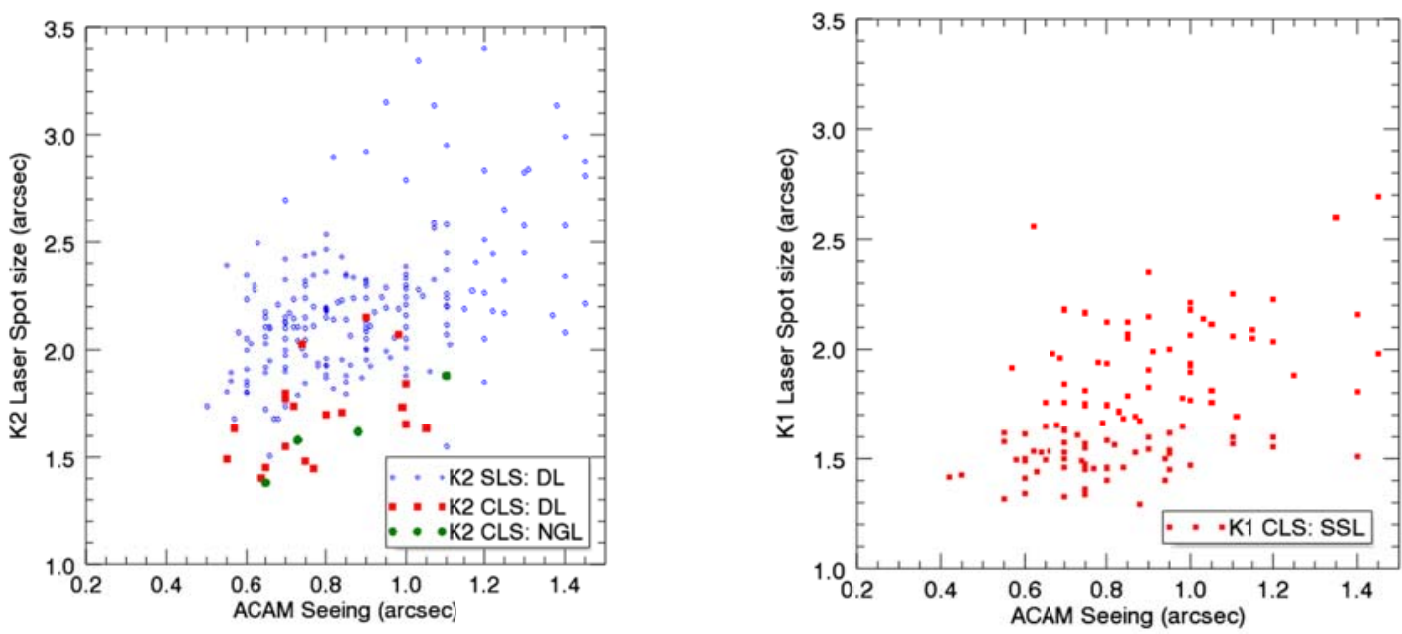

Figure 20: Laser spot size versus ACAM FWHM seeing for NGL and DL at CLS and SLS configurations (left). Keck I spot size data for the solid state laser with CLS (right). 
The mean spot elongation of the CLS is $12 \%$ versus $\sim 40 \%$ for the SLS as seen in Figure 21 . The CLS mean value is a combination of both the NGL and DL with the CLS. For the NGL CLS alone, the ratio is closer to 1 . The difference between the DL and NGL CLS is due to the difference in the laser beams and possible misalignment of powered optics in the beam train. Measurements of the NGL symmetry using a Shack Hartmann camera at the output of the laser showed $2 \%$ asymmetry (Figure 9 ).
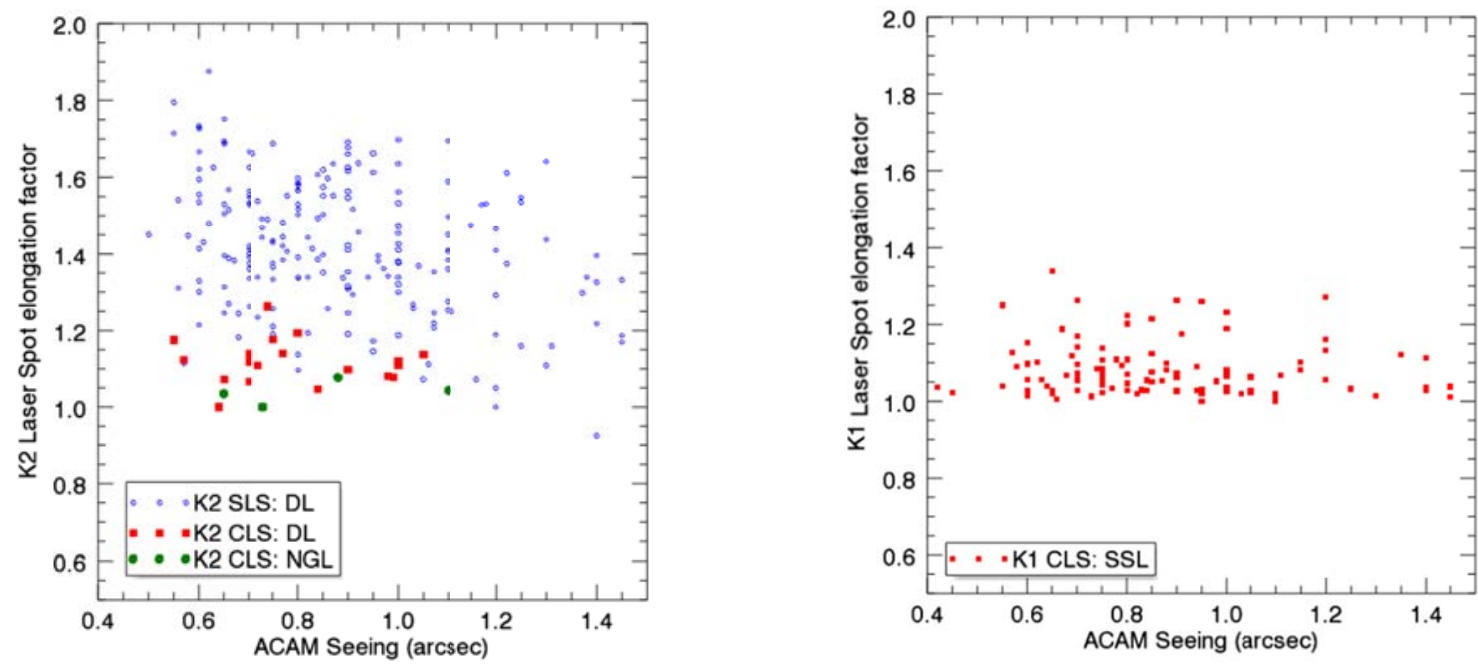

Figure 21: Laser spot elongation versus relative seeing. Left: Keck II SLS and CLS with the dye laser and CLS with the NGL. Right: Keck I CLS with the solid state laser.

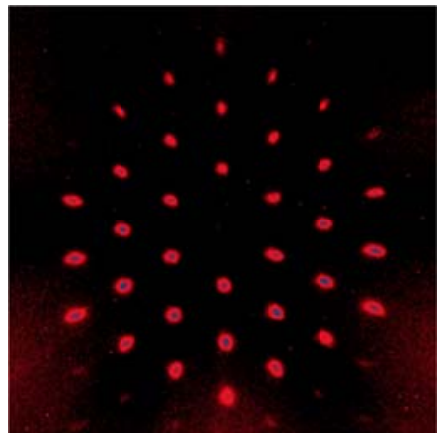

Figure 22: LGS images from the 36 unstacked primary mirror segments of the Keck telescope for the CLS with NGL (the ghost images are caused by a dichroic beam-splitter; variation in the top to bottom intensity is due to background noise on the detector). The center six segments are closest to the laser projector and show the least amount of perspective elongation due to the thickness of the sodium layer.

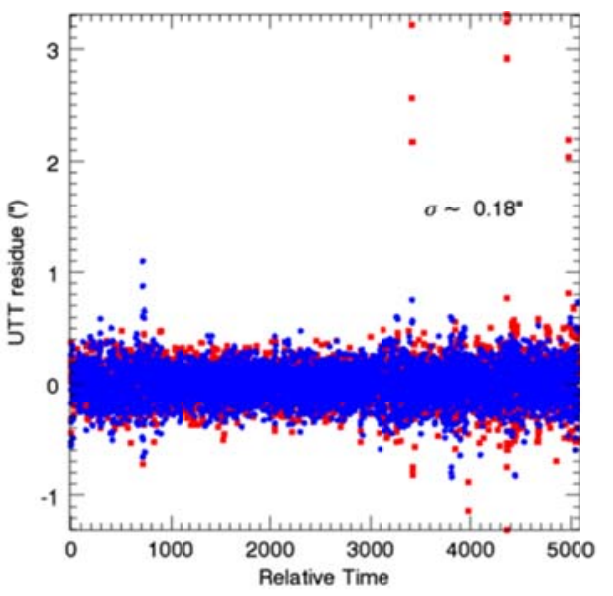

Figure 23: UTT residue measured on UT Dec. $27,2015$.

\section{LGS AO PERFORMANCE}

\subsection{Uplink tip-tilt (UTT) and pointing}

The UTT stage in the laser beam train performs both a UTT and pointing offset function. The UTT stage and a BM5 voice coil mirror provide coordinated laser steering moves of up to $\sim 80^{\prime \prime}$ off-axis. Half of this range is nominally taken up by pointing offsets to account for elevation flexure and the launch telescope's physical pointing offset. This limits the available motion in the launch telescope's unvignetted field of view to $\pm 45^{\prime \prime}$. Better biasing of the error in elevation and better physical alignment of the launch telescope can improve this range for off-axis steering. For UTT performance, the stage is well within its range to address the UTT residue which is about 0.18" (Figure 23). 


\subsection{NIRC2 LGS AO performance}

Recent NGL measurements taken during one of the commissioning runs, along with previous dye laser CLS and SLS data (since Aug. 2010) are provided in Figure 24. The long term data is taken at the start of the night during laser checkout with the telescope near zenith. Overall, the Strehl ratio and FWHM of the NGL CLS are comparable to that of the SLS and CLS with the dye laser. With this minimal NGL data set no definitive performance improvement can be shown. Strehl ratio improvements are expected from the reduced measurement and bandwidth errors due to higher sodium return.
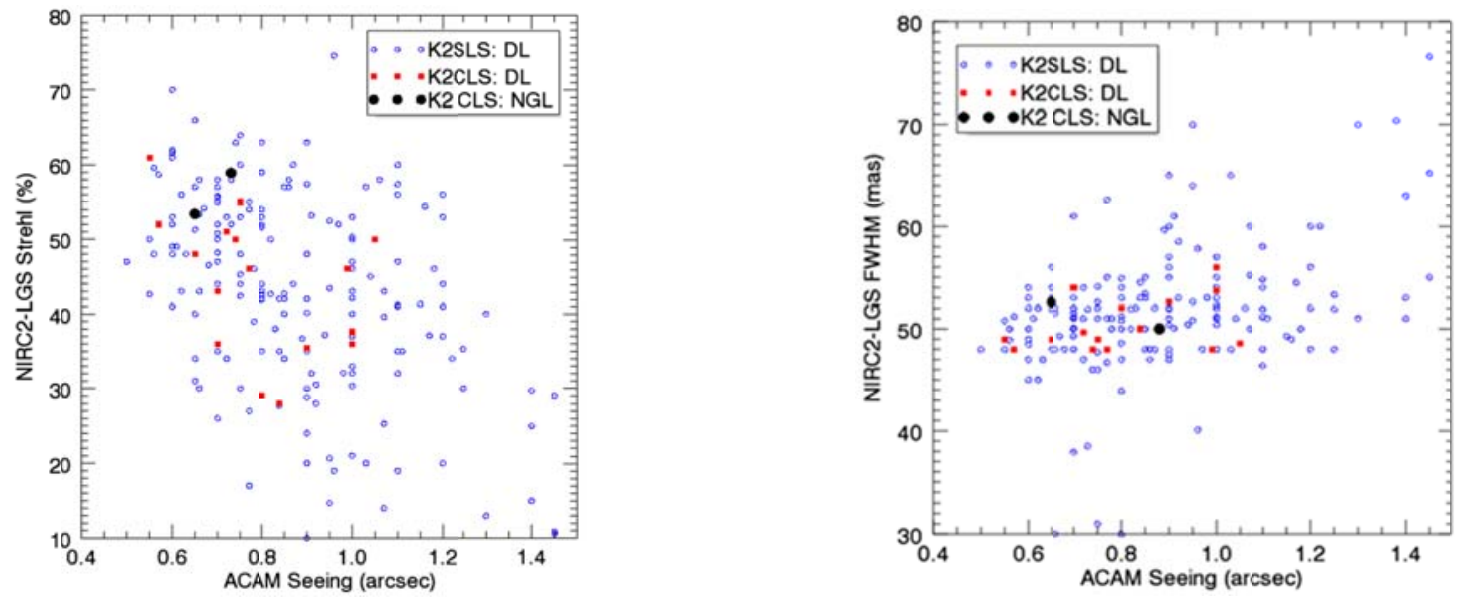

Figure 24: NIRC2 LGS Br $\gamma\left(\lambda_{\text {eff }}=2.1685 \mu \mathrm{m}\right)$ Strehl ratio (left) and FWHM (right) using CLS and SLS.

The reduction of the spot size from SLS to CLS should provide a factor of 1.32 reduction in measurement error; however, the reduced CLS throughput increases the error by a factor of 1.26. For the CLS dye laser to SLS dye laser, the resulting improvement is negligible since the overall wavefront error is large $(370 \mathrm{~nm})$ compared to the measurement error of $150 \mathrm{~nm}$. With the NGL CLS return being 12 times brighter than the SLS dye laser, the combination of the reduced spot size and the improved return should improve the measurement and bandwidth errors. The dye laser CLS and NGL CLS Strehl ratio and FWHM performance at $\operatorname{Br} \gamma\left(\lambda_{\text {eff }}=2.17 \mu \mathrm{m}\right)$ are compared in Figure 24, Figure 25, and Figure 26 in various seeing conditions. The NGL Br $\gamma$ Strehl ratio under excellent seeing conditions nearly reaches $65 \%$. However, there is significant scatter in the Strehl ratio that may be related to the low bandwidth truth wavefront sensor (LBWFS) since the LBWFS was recently replaced due to a failure and was not yet optimized.
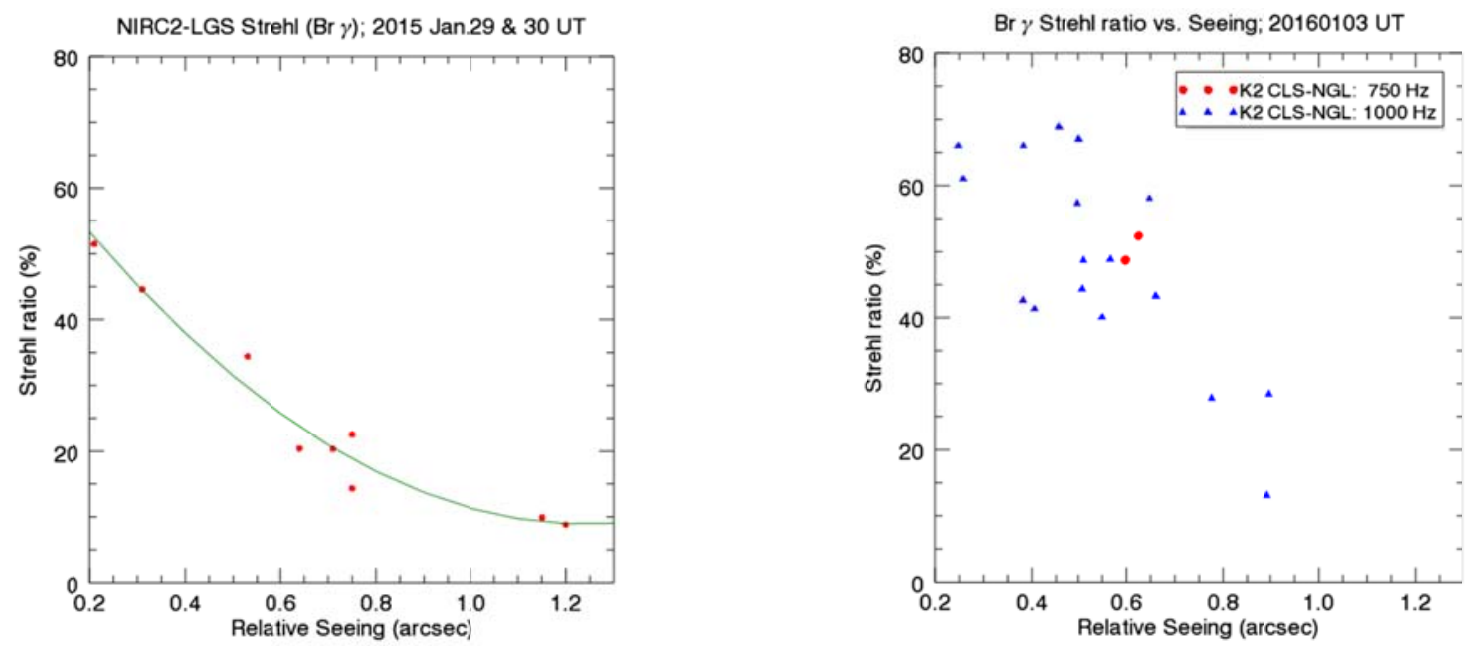

Figure 25: NIRC2 LGS Br $\gamma$ Strehl with the dye laser (left) and the NGL (right) against relative seeing. The dye laser measurements were taken at $750 \mathrm{~Hz}$. 

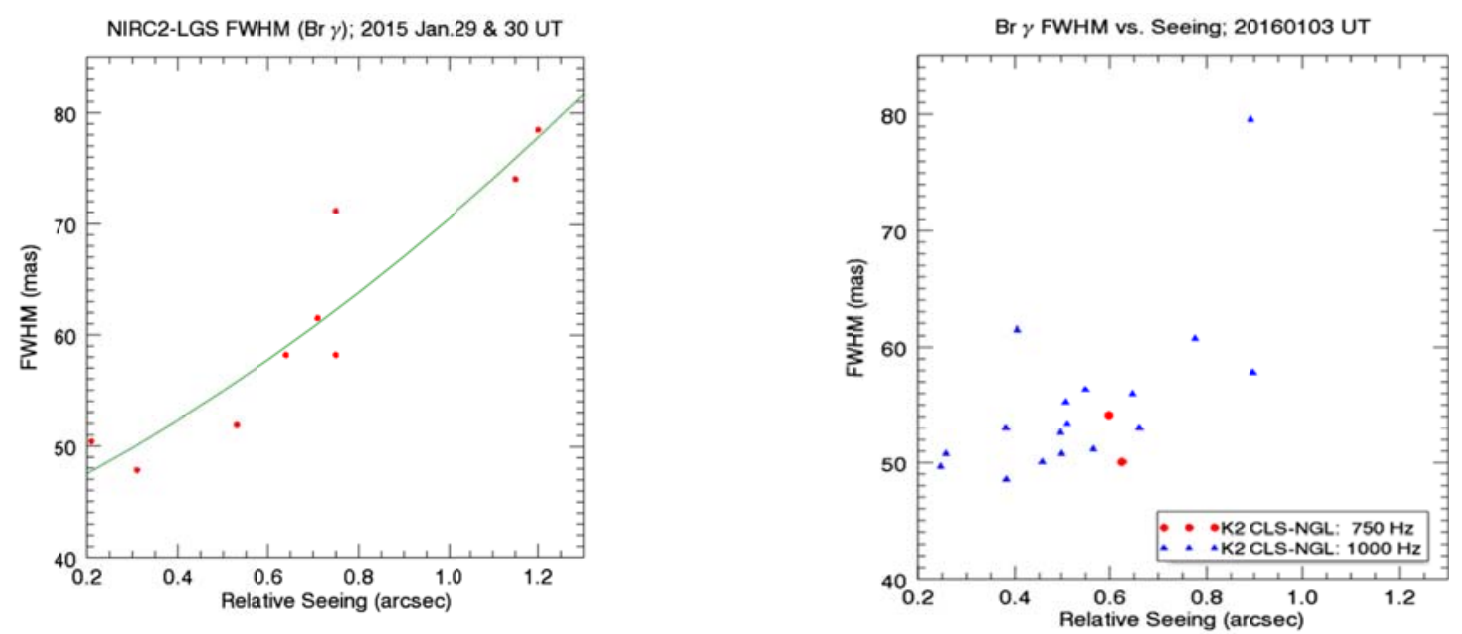

Figure 26: NIRC2 LGS Br $\gamma$ FWHM with the dye laser (left) and the NGL (right) against relative seeing.

Since the laser's output power can be easily changed, a test was conducted on 2016_01_28 UT to determine how the laser return impacts the performance. The frame rate was set to $1 \mathrm{kHz}$ while the laser's power varied from 16 to $22 \mathrm{~W}$. No noticeable change was seen in the Strehl as well as FWHM. On 2016_01_03 UT, 61\% Strehl and 50 mas FWHM images were achieved at the $16 \mathrm{~W}$ laser power setting. The telescope position was at $140^{\circ}$ azimuth and $82^{\circ}$ elevation with an LGS R magnitude of 8.0 for this test. This confirms the LGSAO system is not limited by laser return at the moment. We intend to improve the $\mathrm{AO}$ system bandwidth to determine if this will allow a performance gain given the high photon return of the NGL.

\subsection{Laser Guide Star AO Science}

Science validation observations have been carried out on two nights. The goal was to observe one or two previously observed targets at similar observational and instrumental settings. We present here one of the best observations taken on Jan. 22 UT using the NIRC2 narrow camera and the CH4-short filter. This object was observed twice previously with the same filter and camera settings. The airmass for these observations were in the range 1.40 to 1.45 . The laser was on-axis for the two LGS observations. Figure 27 shows the reduced images from all three epochs. The performance of the new laser was excellent. Unfortunately, different tip/tilt guide stars were used for these observations - an off-axis tip/tilt star $\mathrm{R}=16.4$ magnitudes at 11 " separation was used for the 2015 April observations, while the on-axis science target itself $(\mathrm{R}=14.6)$ was used for the other two observations. Hence direct comparison of these measurements is not possible.

2015 Apr. 10; LGS (DYE LASER)

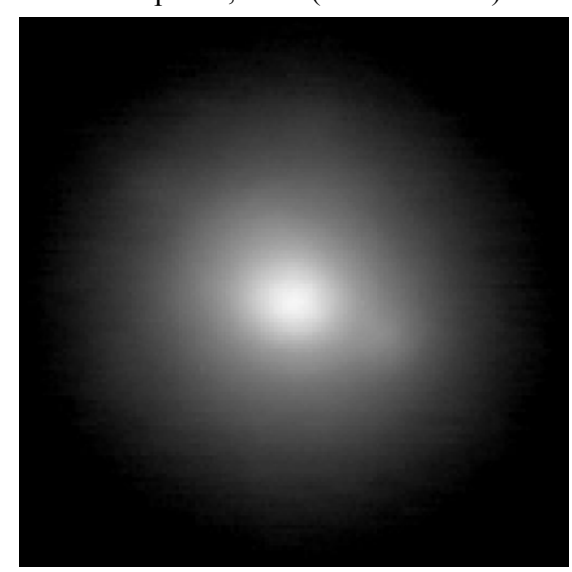

2016 Jan. 22; LGS (NGL)

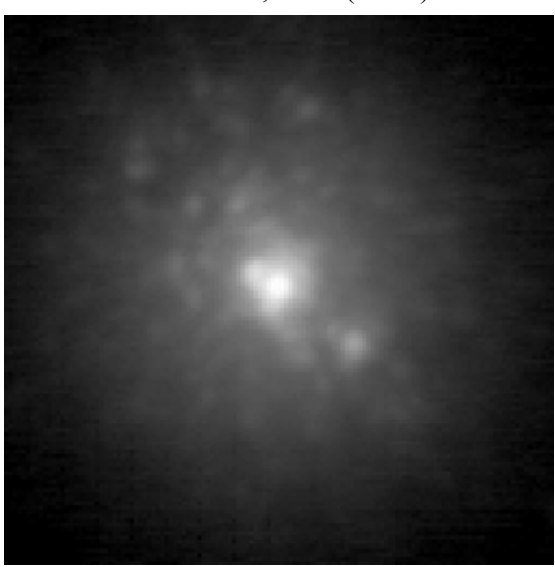

2015 Sep. 23; (NGS)

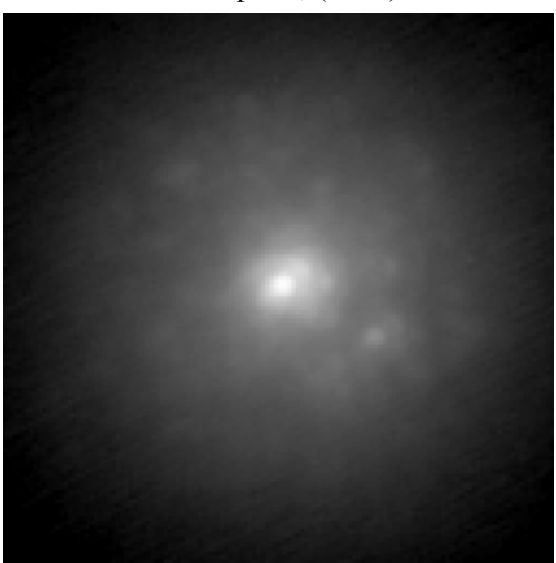

Figure 27: NIRC2 science images of an identical object in similar observing conditions; but with different lasers and with NGS. The left image is with the use of the dye laser with the CLS; center image is with the NGL and CLS; right image is with the use of an NGS for correction. 


\section{CHALLENGES AND OPERATION CONSIDERATIONS}

\subsection{Laser Pointing}

A major challenge for the NGL project was the pointing of the laser onto the wavefront sensor. An initial offset position at telescope zenith is found to align the laser beam to the wavefront sensor's \pm 2 "field of view. A pointing model versus telescope elevation was then developed to correct for flexure. The laser return frequently did not end up on the wavefront sensor even with the pointing model due to hysteresis in the flexure. The root cause of elevation dependent laser pointing is shown in Figure 28. Motion was seen in the telescope's secondary (M2) module, which supports the M2 mirror, the launch telescope, and projection optics. The M2 module defining mechanism to the telescope is unable to define the module properly resulting in flexure and hysteresis. Until this is addressed, an automated search tool backed up by manual hand paddling is used to position the laser return onto the wavefront sensor.
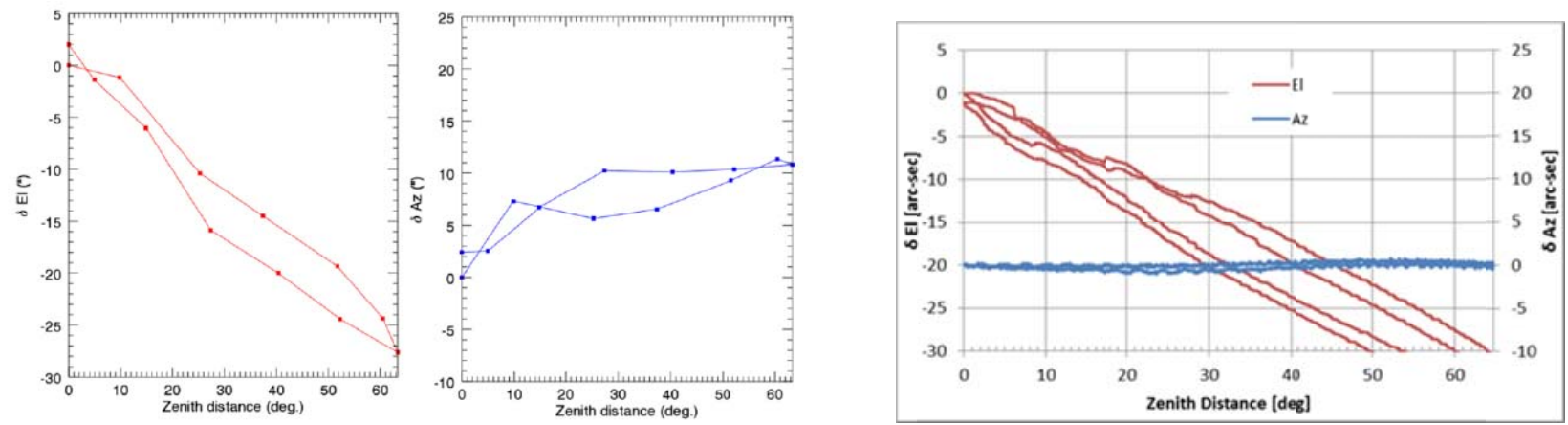

Figure 28: The two plots at left show the laser azimuth and elevation pointing corrections versus zenith angle. The right plot shows the motion of the telescope's M2 module versus zenith angle. The corrections correlate well with the motion of the M2 module.

\subsection{Laser Startup Time and Stability}

The laser can be brought up to full power within minutes if laser internal component temperatures are within acceptable limits. This assumes the system has a valid wavelength calibration. The startup time is much longer if the laser has been powered OFF for 3 days in the Keck dome environment, nominally at $0{ }^{\circ} \mathrm{C}$. During the period the laser is powered OFF, the laser's electronics cabinet internal temperature drops to the ambient dome temperature. Once laser power is enabled, the system sits in the STBY mode waiting for internal component temperatures to rise. The components most isolated due to stability concerns are also the components that require the most time to thermalize, the wave meter and the seed laser. The thermal load within the EC is $<200 \mathrm{~W}$ in this mode when the diodes are OFF. The diodes generate the largest heat load dissipated by the coolant. Heat is also provided minimally by the coolant pump in the HEX. Figure 29 shows the need for the laser to stay in this mode for $6+$ hours to reach acceptable temperature levels before the transitioning to the ON mode. Internal interlocks prevent the laser from turning $\mathrm{ON}$.

There are two heaters in the laser system to reduce the startup time even in a cold soak situation. A heater located in the LH prevents temperatures below $14{ }^{\circ} \mathrm{C}$ to avoid thermal shock of optical components. A second, larger heater is located in the HEX to maintain the laser coolant near $12{ }^{\circ} \mathrm{C}$

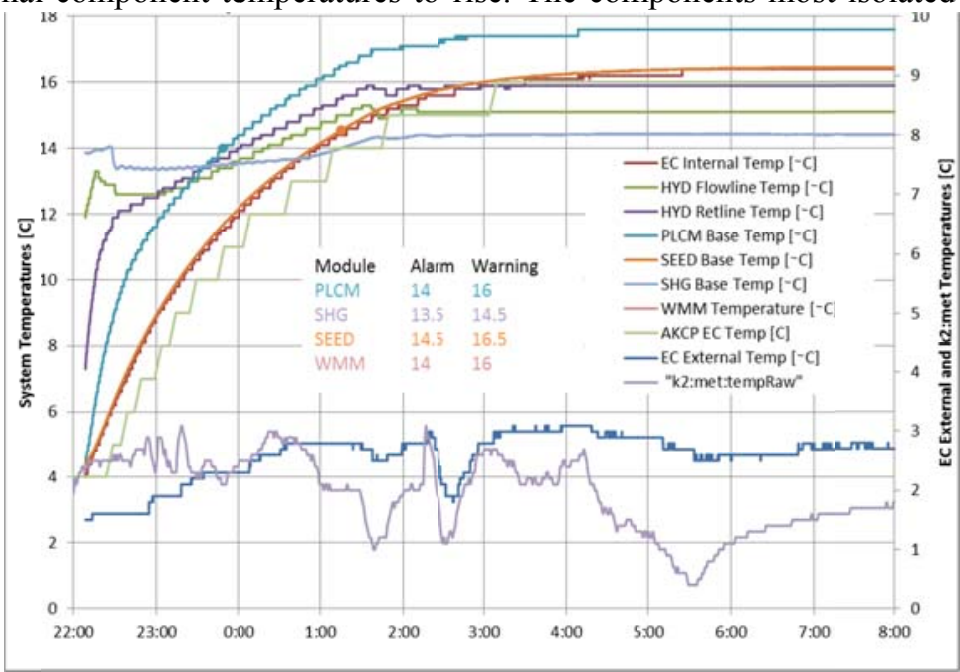

Figure 29: Laser internal component and external temperatures while in the STANDY mode starting from OFF. Data was taken on Mar 16, 2016. 
while it is OFF. If the coolant is not warm enough, it has the undesirable effect of cooling the system as coolant is flowed through the system. The combination of these two heaters reduces the time to turn the laser ON to 6 hours. Once the diodes are turned $\mathrm{ON}$, it takes an additional hour for all system temperatures to stabilize.

\subsection{Ongoing Health Checks and Preventative Maintenance}

A key advantage of the TOPTICA laser is its ability to continuously monitor system health during operations. More intensive offline health checks are also run periodically. The combination of these real time and offline health checks inform the user of any impending problems and to plan for appropriate actions. An example of this would be a possibly failing diode requiring attention or excessive stimulated Brillouin scattering in the fiber. Laser operational parameters are logged by the laser and can be retrieved remotely for analysis. Preventative maintenance is completed by the laser technician for both the laser itself and infrastructure equipment. Beam train optics maintenance, coolant filters and levels, vibration isolators, safety system interlocks, and generally cleanliness are verified periodically.

\subsection{Operations Model and Staffing}

To overcome the cold start warm up period, power is turned ON to the system one day before the laser run allowing the laser to athermalize. Power is applied to the laser in the STANDBY state; but not to the diodes. This significantly reduces the startup of the laser from hours to minutes as shown in Table 2. For a normal LGSAO night, the technician turns on the laser OBSERVATION mode in the afternoon to ensure the laser's functionalities and verify the CLS alignment. These checks ensure the laser and the beam train devices are operational and the laser beam looks acceptable on the beam train cameras. On the first day of a LGSAO run, the beam is propagated onto the dome wall to check for symmetry. This verifies the beam train has not been compromised by activities on the telescope between laser runs. The laser wavelength is calibrated as needed based on run time. Currently, calibrations are completed approximately once per month; longer term historical data may dictate a different frequency. The laser is returned to a READY state after the checkout to reduce diode usage and the laser is turned back to OBSERVATION mode just minutes before propagating on-sky. The laser is put back into its STBY mode via a script at the end of the observing night. Other afternoon activities include verification of systems related to on-sky propagation such as the aircraft detection system to simulate an oncoming aircraft, the laser traffic control system, and the satellite avoidance tool. Most of the above activities can be done remotely.

The estimated effort to operate the laser for 140 nights per year is $\sim 0.25$ Full Time Equivalents (FTE). Additional effort is spent to monitor laser parameters in this initial phase of operations to ensure there is no degradation of the system. In addition, a technician spends $\sim 0.25$ FTE to support maintenance of the laser and associated subsystems.

\subsection{Operations Uptime}

The TOPTICA laser operated for 121 hours between April 15 and May 23, 2016 for science operations. Since this period, there has only been one incident of failure related to the laser which resulted in 5.6 hours of lost time or $4.6 \%$ of the observing time. The NGL system, including the laser, has a lost time percentage of $4.8 \%$ compared to the requirement of $1.5 \%$. This single incident was due to an intermittent failure of the wave meter used to lock the seed laser wavelength. The wave meter was unable to sense the wavelength, causing erratic behavior and a significant power drop in the second harmonic generation cavity; the wave meter started working again without any changes. The wave meter is now carefully monitored for any future issues. A work around has also been developed with TOPTICA to operate the laser in open loop if necessary for future failures. A sodium cell in the LTE can be used to optimize the wavelength manually.

\section{ADAPTIVE OPTICS UPGRADES AND ACTIVITIES}

Keck Observatory's 2016 scientific strategic plan continues to emphasize the importance of high spatial resolution science. The Keck AO systems have been developed for high Strehl ratios with good sky coverage. This direction will continue to be pushed with higher Strehl ratios and higher sky coverage. The new Keck II laser and laser center launch system were developed in support of decreasing the high order measurement and bandwidth errors and hence increasing the Strehl ratio. The Keck I near-infrared tip-tilt sensor was developed in support of higher sky coverage and we are continuing to develop this sensors capabilities into areas such as multiple tip-tilt stars to reduce tip-tilt anisoplanatism (Femenia-Castella et al., [16]) and the use of focal plane sensing to provide fast focus measurements (Plantet et al., [17]). 
The next major proposed steps for the Keck II LGS AO system build on the new laser by splitting the laser into multiple beacons in order to perform tomography and thereby increase the Strehl ratio by reducing the focal anisoplanatism error, and on the Keck I tip-tilt sensor by implementing a Keck II near-infrared tip-tilt sensor to improve sky coverage (Wizinowich et al., [18]).

Quantitative high spatial resolution science also relies on knowledge of the point spread function (PSF). Two PSF reconstruction (PSF-R) demonstration efforts using Keck AO science data, AO telemetry and atmospheric profiler data are nearing fruition. The on-axis PSF-R effort is reported on by Ragland et al. [19]. The off-axis PSF-R efforts are reported on by Witzel et al. [20], Do et al. [21] and Sitarski et al. [22]. The next step, planned to begin in 2017, is to turn these demonstrations into a PSF-R facility that provides a PSF estimate anywhere in the science field for every AO science observation.

Keck AO with the NIRC2 and OSIRIS science instruments has proven to be very useful for exoplanet imaging and characterization. The role of Keck AO in this field has recently been enhanced with the installation and commissioning of an L-band vortex coronagraph focal plane mask in the NIRC2 science instrument (Femenia-Castella et al., [23]). The next step being undertaken as part of a phased approach to implementing the Keck Planet Imager and Characterizer (KPIC; Mawet, [24]) will be to integrate a single mode fiber injection unit on the Keck II AO bench to feed the NIRSPEC science instrument; the fiber injection unit includes a pupil plane where apodizers can be installed (Ruane et al., [25]). A proposal for a near-infrared pyramid wavefront sensor has been submitted that would support NGS AO science observations with both NIRC2 and the fiber injection unit (Wizinowich, [26]). A proposal for a coronagraph module has also been submitted which would include a MEMS deformable mirror in a pupil plane and a focal plane coronagraph mask, which would be installed just before the fiber injection unit.

The OSIRIS science instrument has recently been upgraded by replacing its spectrograph detector with an H2RG detector. The OSIRIS imager optical system and detector will also be upgraded in early 2017 (Witzel et al., [27]). A NIRSPEC detector upgrade is also in progress and a proposal to implement a precision radial velocity (PRV) capability with NIRSPEC has been submitted. The NIRSPEC PRV mode would be fed with a single mode fiber from the Keck II AO system; both NGS and LGS AO observations would be supported.

A number of modest AO performance and maintenance upgrades are underway in support of all AO observations. Semistatic low order structure in the first airy ring of the AO-corrected PSF has recently been traced to correlated low order piston errors in the segmented primary mirror and we intend to reduce or characterize this contribution to the PSF. The Keck AO benches tend to operate 5 to $10{ }^{\circ} \mathrm{C}$ warmer than the dome temperature and we are working to reduce the bench temperatures in order to reduce the resultant emissivity. The Keck II low bandwidth (truth) wavefront sensor camera has been recently replaced with a faster readout camera and the $5 \times 5$ mode of this system will be made more fully operational to allow for faster updates. The Keck II AO acquisition camera will be replaced with a newer camera in 2017 and we will use this opportunity to look at also making this a science camera.

The Observatory also intends to begin studying the scientific utility of ground layer AO for Keck science and to determine the feasibility of using existing multi-object spectrographs (DEIMOS, LRIS and/or MOSFIRE) with this mode.

\section{CONCLUSIONS}

The operational use of LGS for AO has been a great success and driver for science and publications at the W. M. Keck Observatory in the last decade. The addition of a Raman fiber laser, along with the recent center launch system, will allow continued success as the observatory moves toward its goal of higher Strehl ratios and higher sky coverage. The laser has demonstrated its ability to operate as a facility class instrument and the sodium returns are matching well with the Bloch Equations to first order and with other observatories using similar format lasers. With the higher sodium returns, the LGSAO system's measurement and the bandwidth errors can be reduced; the limiting factor is in the wavefront sensing and controller and not from sodium returns. The high return also positions WMKO to take the next step of using multiple LGS for laser tomography.

The TOPTICA laser also simplifies the laser operations model for observatories. The efficiency gained by minimizing infrastructure and manpower requirements allows the laser to behave as another typical instrument, unlike its predecessors. The minimal time frame to start the laser at thermal equilibrium and ease of operations will also assist the observatory in supporting time domain astronomy and unattended night time operations. 


\section{ACKNOWLEDGEMENTS}

The development of the Keck II next generation laser is made possible through the support of the Gordon and Betty Moore Foundation, the W. M. Keck Foundation, the Bob \& Renee Parsons Foundation, Friends of W. M. Keck Observatory, and the Thirty Meter Telescope Project.

The development of the Keck II center launch facility is made possible in part by the support of the National Science foundation, and the material in this paper describing this facility is based in part upon work supported by the National Science Foundation under Grant No. AST- 0923593.

We would like to thank the Thirty Meter Telescope Project for funding Dr. Kai Wei to work on the new Keck II laser facility. We would like to acknowledge the technical contributions of Liz Chock, Randall Campbell, James E. Lyke, Matthias Schoeck and Will Best. We would like to thank the NGL science team of Andrea Ghez, Mike Liu and Claire Max. We would also like to thank the laser teams from TOPTICA AG Photonics, MPBC, and the European Southern Observatory.

The data presented herein were obtained at the W. M. Keck Observatory, which is operated as a scientific partnership among the California Institute of Technology, the University of California, and the National Aeronautics and Space Administration. The Observatory was made possible by the generous financial support of the W. M. Keck Foundation.

The authors wish to recognize and acknowledge the very significant cultural role and reverence that the summit of Mauna Kea has always had within the indigenous Hawaiian community. We are most fortunate to have the opportunity to conduct observations from this mountain.

\section{REFERENCES}

[1] Wizinowich, P. et al., "First Light Adaptive Optics Images from the Keck II Telescope: A New Era of High Angular Resolution Imagery," PASP 112, 315-319 (2000).

[2] Friedman, H. et al., "Design and performance of a laser guide star system for the Keck II telescope," SPIE Proc. 3353, 260 (1998).

[3] Wizinowich, P., "Astronomical Science with Adaptive Optics at W. M. Keck Observatory," PASP 125, 798-808 (2013).

[4] Lee, I. et al., "20 W and $50 \mathrm{~W}$ guidestar laser system update for the Keck I and Gemini South telescopes," SPIE Proc. 7015, 70150N (2008).

[5] Friedenauer, A, et al., "RFA-based 589nm guide star lasers for ESO VLT: a paradigm shift in performance, operational simplicity, reliability and maintenance," SPIE Proc. 8447, 84470F (2012).

[6] Chin, J. et al., "Laser Guide Star Facility Developments at W. M. Keck Observatory," SPIE Proc. 9148, 914808 (2014).

[7] Chin, J. et al., "Keck I Laser Guide Star Adaptive Optics System,” SPIE Proc. 8447, 84474F (2012).

[8] Wizinowich, P. et al., "W. M. Keck Observatory's next generation adaptive optics facility," SPIE Proc. 7736, 19 (2010).

[9] LeMignant, D. et al., "Keck Adaptive Optics Note \# 337 Gemini-Keck Star Wars: Keck Perspective," June 29 2005.

[10] Neyman, C. and Chin, J., "Keck Adaptive Optics Note \# 536 Keck I LGSAO System Requirements Flowdown," June 202007.

[11] Bonaccini, D. et al., "LGS field experiments at Observatorio de el Teide," EWASS (2015).

[12] Holzlöhner R. et al., "Optimization of CW Sodium Laser Guide Star Efficiency,” A\&A 510, 0004-6361 January, (2016).

[13] Holzlöhner, R. et al., "Laser Guide Star Flux Simulations based on Observed Sodium Density Profiles," SPIE Proc. 7736, 773615 (2010).

[14] Roberts, L. et al., "Measurements of Mesospheric Sodium Abundance above the Hawaiian Islands," ASP 119, 787792, July (2007). 
[15] Ragland, S. et al., "Recent Improvements to the Keck II Laser Guide Star Facility," Proceeding for AO4ELT Conference (2016).

[16] Femenia-Castella, B. et al., "Status and new developments with the Keck I near-infrared tip-tilt sensor," SPIE Proc. 9909, (2016).

[17] Plantet, C. et al., "LIFT on Keck: analysis of performance and first experimental results," SPIE Proc. 9909, (2016).

[18] Wizinowich, P. et al., W. M. Keck Observatory's next generation adaptive optics facility. SPIE Proc. 7736-19 (2010).

[19] Ragland, S. et al., "Point spread function determination for Keck adaptive optics," SPIE Proc. 9909, (2016).

[20] Witzel G. et al., "The AIROPA software package: milestones for testing general relativity in the strong gravity regime with adaptive optics," SPIE Proc. 9909, (2016)

[21] Do, T. et al., "PSF Reconstruction for the Keck integral-field spectrograph OSIRIS," SPIE Proc. 9909, 2016.

[22] Sitarski, et al., "Astrometry with spatially variable PSFs: instrumental field-dependent aberrations," SPIE Proc. 9909, (2016).

[23] Femenia-Castella, B. et al., "Commissioning and first light results of an L'-band vortex coronagraph with the Keck II adaptive optics NIRC2 science instrument.” SPIE Proc. 9909, (2016).

[24] Mawet, D., "Keck planet imager and characterizer (KPIC): concept and phased implementation," SPIE Proc. 9909, (2016).

[25] Ruane, J. et al., "Advanced coronagraph designs for segmented aperture telescopes," SPIE Proc. 9912-91, (2016)

[26] Wizinowich, P. et al., "Near infrared wavefront sensing," SPIE Proc. 9909, (2016)

[27] Witzel, G. et al., "Motivation for and design of a new imaging camera for the OSIRIS instrument at Keck," SPIE Proc. 9908, (2016). 